NBER WORKING PAPER SERIES

\title{
GLOBALIZATION AND PANDEMICS
}

\author{
Pol Antràs \\ Stephen J. Redding \\ Esteban Rossi-Hansberg \\ Working Paper 27840 \\ http://www.nber.org/papers/w27840
}

\author{
NATIONAL BUREAU OF ECONOMIC RESEARCH \\ 1050 Massachusetts Avenue \\ Cambridge, MA 02138
}

September 2020, Revised November 2022

\begin{abstract}
We thank the editors, Pierre-Oliver Gourinchas and Arnaud Costinot, and four anonymous referees for helpful comments and suggestions. We are grateful to Lorenzo Caliendo, Rob Feenstra, Hiau Looi Kee, John Romalis, and Alan Taylor for their help with data. We also thank Elena Aguilar, Maxim Alekseev, Gordon Ji, Daniel Ramos, Max Schwarz, Marcos Sora, Sifan Xue, Sean Zhang, and Shuhan Zou for excellent research assistance. We have received valuable comments from Andy Atkeson, Elhanan Helpman and conference and seminar participants at the Banque de France, CEPR, Geneva, Harvard University, IATRC, IMF, NBER, NYU, Princeton University, Purdue University, Sardinia Empirical Trade Conference, Stanford University, St. Louis Fed, University of Southern California, Virtual Trade and Macro Seminar, and Yale. All errors, opinions and omissions are our own. The views expressed herein are those of the authors and do not necessarily reflect the views of the National Bureau of Economic Research.
\end{abstract}

NBER working papers are circulated for discussion and comment purposes. They have not been peer-reviewed or been subject to the review by the NBER Board of Directors that accompanies official NBER publications.

(C) 2020 by Pol Antràs, Stephen J. Redding, and Esteban Rossi-Hansberg. All rights reserved. Short sections of text, not to exceed two paragraphs, may be quoted without explicit permission provided that full credit, including $(\odot$ notice, is given to the source. 
Globalization and Pandemics

Pol Antràs, Stephen J. Redding, and Esteban Rossi-Hansberg

NBER Working Paper No. 27840

September 2020, Revised November 2022

JEL No. F1,F2,F4,F6,I1

\begin{abstract}
$\underline{\text { ABSTRACT }}$
We propose a theory of the relationship between globalization and pandemics. We start by documenting the importance of international trade for the diffusion of infections in several pandemics throughout history and by showing that trade and travel are closely intertwined. Motivated by this evidence, we build a framework in which business travel facilitates trade according to a constant elasticity gravity equation mediated by mobility frictions. In turn, travel leads to human interactions that transmit disease, as in the Susceptible-Infected-Recovered (SIR) model. We highlight three novel interactions between these two mechanisms. First, trademotivated travel generates an epidemiological externality across countries. Therefore, reductions in international frictions affect the evolution of the epidemic in each country, and the condition for a pandemic to occur. Second, if infections lead to deaths, or reduce individual labor supply, we establish a general equilibrium social distancing effect, whereby increases in relative prices in unhealthy countries reduce travel to those countries. Third, if agents internalize the threat of infection, we show that their behavioral responses lead to a reduction in travel that is larger for higher-trade-cost locations, and hence leads to an initial fall in the ratio of trade to GDP in the early stages of the epidemic, before a subsequent recovery.
\end{abstract}

Pol Antràs

Department of Economics

Harvard University

1805 Cambridge Street

Littauer Center 207

Cambridge, MA 02138

and NBER

pantras@fas.harvard.edu

Stephen J. Redding

Department of Economics \&

School of Public and International Affairs

Princeton University

Princeton, NJ 08544

and CEPR

and also NBER

reddings@princeton.edu
Esteban Rossi-Hansberg

The Kenneth C. Griffin Department of Economics University of Chicago

5757 S. University Avenue

Chicago, IL 60637

and CEPR

and also NBER

rossihansberg@uchicago.edu 


\section{Introduction}

Throughout human history, globalization and pandemics have been closely intertwined. The Black Death arrived in Europe in October 1347 when twelve trading ships from the Black Sea docked at the Sicilian port of Messina - the word quarantine originates from the Italian word for a forty-day period of isolation required of ships and their crews during the Black Death pandemic. Much more recently, on January 21, 2020, the first human-to-human infections of Covid-19 in Europe are presumed to have taken place in Starnberg, Germany, when a local car parts supplier (Webasto) organized a training session with a Chinese colleague from its operation in Wuhan, China. Examples of disease transmission from the travel induced by international trade abound.

In this paper, we develop a theoretical framework to analyze the relationship between globalization and pandemics. Our framework combines two core mechanisms from economics and epidemiology. First, travel between countries transmits disease through human interaction as in the Susceptible-Infected-Recovered (SIR) model. Second, international trade is facilitated by business travel between countries according to a conventional gravity equation partly mediated by mobility frictions. We show that these core mechanisms are not only empirically relevant, but they interact in systematic ways that lead to novel insights on the interaction between international trade, business travel, and the dynamics of infections during pandemics.

We begin by providing empirical evidence that international trade affects the transmission of disease. We examine the diffusion of three different infectious diseases at different points in history: (i) the medieval plague; (ii) the 1957-8 influenza; (iii) Covid-19. For all three infectious diseases, we show that the speed of diffusion of each disease increases with measures of international trade links. Furthermore, we continue to observe this positive relationship even after controlling for geographical distance from the first disease outbreak.

We next provide empirical evidence that international trade speeds the transmission of disease through the international travel that it induces. First, we use additional data that are available for Covid-19 to show that the relationship between disease diffusion and trade ceases to be statistically significant once we control for total arrivals and departures of people (including migration, tourism and business travel), as expected if the mechanism is international travel. Second, we establish a strong positive and statistically significant correlation between bilateral travel and trade. Third, we show that we continue to observe this relationship if we focus on the variation in bilateral trade predicted by bilateral trade policies, as measured by tariffs. Fourth, we summarize the results of recent studies that have used quasi-experimental variation to provide evidence of a causal relationship between travel and trade.

Motivated by these empirical findings, we develop a theoretical model of globalization and pandemics that incorporates a gravity equation for trade and travel alongside a SIR model of disease diffusion. We consider a setting in which agents in each country consume differentiated varieties and choose the measure of these varieties to source from home and abroad. In our baseline model, agents must travel to source each variety from home and abroad, which involves both a fixed cost of meeting with other agents and a variable cost of shipping varieties between countries. 
When traveling to source varieties, agents are exposed to risk of disease transmission. If a healthy (susceptible) agent meets an infected agent, she becomes infected with a constant probability (the contact rate). Once infected, there is a constant probability that an agent recovers from the disease (the recovery rate). We allow these contact and recovery rates to differ across countries. ${ }^{1}$ Although we interpret this travel between countries as business trips, another natural interpretation is tourism, which involves travelling between countries to consume services.

We begin by abstracting from any effect of the infection on the probability of death or worker productivity, and hence from any behavioral responses. The resulting open economy disease dynamics take a similar form as in existing multi-group SIR models. However, a key difference from those existing models is that bilateral interactions between countries are endogenously determined by international trade and travel frictions. We demonstrate the presence of an epidemiological externality between countries in the open economy equilibrium. A sufficient condition for a pandemic to occur is that any country has a reproduction number based on domestic interactions alone of greater than one, where the intensity of these domestic interactions differs between the open and closed economies. Therefore, even if one country has the disease under control based on its domestic reproduction number, it may not escape a pandemic if its trade partners do not. We show that reductions in international trade and travel frictions can either increase or decrease the range of parameters where a pandemic occurs and the severity of a pandemic when it does occur. On the one hand, this international integration weakly raises the net benefits of interactions, which tends to increase the overall number of interactions, and hence raise the likelihood and severity of a pandemic. On the other hand, this international integration induces agents to substitute foreign for domestic interactions. Depending on the domestic and foreign disease environments, this substitution can either raise or reduce the likelihood and severity of a pandemic.

To develop these insights as clearly as possible, we keep our baseline model stylized. In the Appendix, we demonstrate the robustness of these results to a large number of extensions. For example, we relax the assumption that travel is required to source each variety by allowing agents to choose between different technologies for sourcing varieties: in-person versus remote trade. Inperson trade is assumed to involve, on average, a higher fixed cost. We also develop a dynamic extension in which agents only travel to form new trading relationships. Agents can source varieties within existing trading relationships without travelling, but these existing relationships break down stochastically. In other generalizations, we demonstrate the robustness of our results to alternative specifications of the fixed cost of sourcing varieties, to the sourcing of intermediate inputs, and to frameworks featuring multiple sectors, multiple countries, scale economies, and imperfect competition. We show that all of our main theoretical results continue to hold in these generalizations of our framework.

We next allow the disease to affect the probability of death and the labor supply of infected workers, but initially continue to assume that agents remain unaware of the source of the infec-

\footnotetext{
${ }^{1}$ Differences in contact and recovery rates across countries stand in for differences in local culture (e.g., mask wearing), administrative capacity (e.g., contact tracing systems), and medical technology (e.g., available medical treatments).
} 
tions, which implies no individual-level behavioral responses. Nevertheless, deaths and lower worker productivity reduce aggregate labor supply. Therefore, a country with a more unhealthy disease environment experiences a reduction in its relative supply of labor, and hence an increase in its relative wages. This increase in the country's relative wage raises the relative price of its goods, which reduces travel to that country, in what we term a "general equilibrium social distancing" effect. The timing of these changes in relative labor supply depends on the extent to which the disease affects mortality versus worker's labor supply while infected. Furthermore, which country has the more unhealthy disease environment can change over the course of the pandemic, if the timing of the disease outbreak differs across countries. Additionally, if the disease affects worker's labor supply, and workers self-isolate while infected, their interactions with other agents fall proportionately with their labor supply, which flattens the infection curve and reduces the total number of infections. We highlight that these predictions for the impact of pandemic-induced changes in labor supply on wages find empirical support from both the Black Death and 1918 Influenza.

Finally, we consider the case in which the disease affects the probability of death, and agents internalize the threat of infection, and optimally adjust their behavior depending on the observed state of the pandemic. As in recent work (see Farboodi et al., 2021), it proves useful to assume that agents are uncertain about their own health status, and simply infer their health risk from the shares of their country's population with different health status (something they can infer from data on pandemic-related deaths). Technically, this turns the problem faced by agents into a dynamic optimal control problem in which the number of varieties that agents source from each country responds directly to the relative severity of the disease in each country.

As in recent closed-economy models of social distancing (such as Farboodi et al., 2021, or Toxvaerd et al., 2020), these behavioral responses reduce human interactions, and thereby tend to flatten the curve of infections. In contrast to these closed-economy setups, these behavioral responses now have international general equilibrium implications. In both countries, agents skew their interactions away from the relatively unhealthy country, which leads to the largest falls in the ratio of trade to income in the relatively healthier country. This redirection of interactions reduces the relative demand for the unhealthy country's goods, which in turn reduces its relative wage, thereby having the opposite effect to the reduction in its relative labor supply from greater deaths. Depending on the timing of the wave of infections in each country, which country has more infections than the other can again change over the course of the pandemic, thereby reversing this pattern of changes in trade openness and relative wages over time. We show that these behavioral responses lead to a larger reduction in travel for higher-trade-cost locations, which leads to an initially larger fall in the ratio of trade to GDP in the early stages of the pandemic, before its subsequent recovery. This theoretical prediction of a decline in trade relative to GDP receives empirical support from the recent COVID-19 pandemic, where world trade indeed fell faster than world production in the early stages of the pandemic, before later rebounding. More generally, the prediction that behavioral responses lead agents to reduce interactions because of the threat of infection is supported by a range of empirical findings for COVID-19, as we argue at the end of 
Section 6.2.

Our paper connects with several strands of existing research. First, we build on an extensive empirical literature that finds that a constant elasticity gravity equation provides a good approximation to observed spatial interactions, including international trade (e.g., Anderson and van Wincoop 2003, Eaton and Kortum 2002, Arkolakis et al. 2012, Chaney 2014), migration (Kennan and Walker 2011), commuting (Ahlfeldt, et al. 2015, Monte, et al. 2018), and tourism (Morley, et al. 2014). Relative to this existing research, we incorporate a gravity equation for trade and travel alongside a SIR model of disease dynamics. Since travel and disease diffusion are endogenous to trade frictions, the gravity structure of trade determines both whether a pandemic occurs and the severity of the pandemic when it does occur.

Second, we build on the empirical literature on the role of international business travel in greasing the wheels of international trade. A large number of empirical studies find a strong correlation between international travel and international trade, including Kulendran and Wilson (2000), Cristea (2011) and Blonigen and Cristea (2015). More recently, a small number of studies have used micro data and sources of quasi-experimental variation to provide evidence of a causal impact of business travel on trade, including Bernard, Moxnes and Saito (2019), Campante and YanagizawaDrott (2018), Söderlund (2020), and Startz (2021). Relative to this research, we examine the role played by the link between international travel and trade in the transmission of disease.

Third, our paper also builds on the literature developing epidemiological models of disease spread, starting with the seminal work of Kermack and McKendrick (1927, 1932). More specifically, our multi-country SIR model shares many features with multigroup models of disease transmission, as in the work, among others, of Hethcote (1978), Hethcote and Thieme (1985), van den Driessche and Watmough (2002), and Magal et al. (2016). ${ }^{2}$ A key difference is that the interaction between groups is endogenously determined by international trade frictions, which implies that trade costs affect disease dynamics in each country and the world as a whole.

The recent Covid-19 pandemic has triggered a remarkable explosion of work by economists studying the spread of the disease (see, for instance, Fernández-Villaverde and Jones, 2022) and exploring the implications of several types of policies (see, for instance, Alvarez et al., 2021, Acemoglu et al., 2021, Atkeson, 2020, or Jones et al., 2021). Within this literature, a few papers have explored the spatial dimension of the Covid-19 pandemic by simulating multi-group SIR models applied to various urban and regional contexts (see, among others, Argente et al., 2022, Bisin and Moro, 2021, Cuñat and Zymek, 2020, Birge et al., 2020, and Fajgelbaum et al., 2021). Our paper also connects with a subset of that literature, exemplified by the work of Alfaro et al. (2020), Farboodi et al. (2021), Fenichel et al. (2011), and Toxvaerd (2020) that has studied how the behavioral response of agents (e.g., social distancing) affects the spread and persistence of pandemics. Most of this research is concerned with Covid-19 and adopts a simulation approach. In contrast, we develop a theoretical framework that permits an analytical characterization of the relationship

\footnotetext{
${ }^{2}$ See Hethcote (2000) and Brauer and Castillo-Chavez (2012) for very useful reviews of mathematical modeling in epidemiology, and Ellison (2020) for an economist's overview of SIR models with heterogeneity.
} 
between globalization and pandemics. We provide empirical evidence in support of the predictions of this theoretical framework using data from a range of different infectious diseases.

Our work is also related to a large existing empirical literature in epidemiology on the role of global trade and transportation networks in the transmission of infectious disease. This body of research is summarized in Saker et al. (2004) and the National Academies of Science Conference Volume by Institute of Medicine (2006). In a related review of the existing evidence, Tatem et al. (2006) examine five human-infectious diseases (Plague, Cholera, Influenza, HIV and SARS) and four vector-borne diseases (Yellow Fever, Dengue, West Nile Virus, Malaria), and document the role played by international trade in the spread of these diseases around the globe. In the economic history literature, Benedictow (2004), Christakos et al. (2005), Boerner and Severgnini (2014), Yue et al. (2017), and Jedwab et al. (2019) all argue that international trade routes were central to understanding the transmission of the plague through medieval Europe. Kenny (2021) provides an insightful account of the effect of pandemics throughout history.

The rest of the paper is structured as follows. In Section 2, we provide empirical evidence that international trade speeds the transmission of disease and that the mechanism is through the international travel induced by trade. In Section 3, we present our baseline gravity model of travel and trade. In Section 4, we examine the implications of this model for disease dynamics, abstracting from labor supply effects from deaths or reduced worker productivity and from behavioral responses. Globalization plays a central role in shaping the course of the pandemic, because the volume of travel between countries is endogenous to trade and mobility costs. In Section 5, we incorporate labor supply responses to the pandemic, which affect the path of relative wages and thus the volume of travel between countries during the pandemic. In Section 6, we allow for individual behavioral responses motivated by agents adjusting their desired travel behavior in response to their fear of being infected by the disease. We offer some concluding remarks in Section 7. An Online Appendix presents all the proofs and extensions not included in the main text, as well as additional robustness exercises, empirical evidence, data description, and computational algorithms.

\section{Motivating Evidence}

We start by providing new evidence on the link between international trade and disease diffusion and the underlying mechanism connecting international travel and trade. ${ }^{3}$ First, we provide some historical background on globalization and disease diffusion. Second, we document the speed of diffusion for three infectious diseases: the medieval plague, 1957-8 influenza, and Covid-19. Third, we provide evidence on the cross-sectional relationship between the speed of diffusion and international trade for each disease. Finally, we provide evidence on the link between international travel and trade, as the mechanism connecting disease diffusion and trade in our theoretical model.

\footnotetext{
${ }^{3}$ For additional evidence and literature reviews, see Online Appendix G on the link between trade and disease diffusion, and Online Appendix $\mathrm{H}$ on the link between travel and trade.
} 
Historical Background For most of human history, regional and continental populations were relatively isolated from one another. Large-scale improvements in land, sea, and air transportation technologies over the centuries have dramatically increased the globalization of the world economy and the associated movement of people and goods around the world. ${ }^{4}$ Perhaps the most dramatic example of international trade spreading infectious disease comes from Christopher Columbus's discovery of the New World in 1492, during his search for a more direct trade route with China and the Spice Islands. Since the natives of the New World had no accumulated immunity to Old World infectious diseases, such as smallpox, measles, and influenza, the subsequent epidemics of these infectious diseases resulted in mortality rates of up to 80-90 percent. ${ }^{5}$

Disease Diffusion over Time We provide empirical evidence for three quite different diseases at different points in history: (i) The medieval plague; (ii) The 1957-8 influenza; (iii) The Covid19 pandemic. Both the influenza virus and Covid-19 are spread through airborne transmission between people in close proximity. Although debate continues about the exact origin and mode of transmission of the plague, the consensus is that the plague is initiated by the flea-borne bacterium Yersina pestis, which circulates between mammal hosts. ${ }^{6}$

We use data on plague outbreaks across European cities from 1347-1760 and outbreaks of influenza from 1957-8 and Covid-19 from 2019-2020 across countries. The medieval plague first arrived in Europe at Messina in 1347 and then spread along Old World trading routes. The first cases of both 1957-8 influenza and Covid-19 were in China, before spreading around the world. For each disease, we compute an arrival time for each location, as the difference in time between the first outbreak of the disease in that location and its first outbreak anywhere. ${ }^{7}$

In the left panel of Figure 1, we show the distribution of these arrival times in years for the plague across European cities. Given the relatively low economic integration in the medieval period, some cities were infected by the plague early on, whereas others escaped earlier epidemics, only to be infected in a later outbreak. In the right panel of Figure 1, we show the distribution of these arrival times in days for the 1957-8 influenza and Covid-19 pandemics across countries. Again we find that it takes time for each infectious disease to diffuse across countries. But the arrival times are substantially more rapid for the 1957-8 influenza and Covid-19 pandemics, consistent with the much greater integration of the world economy in more recent decades. ${ }^{8}$

Trade and Disease Diffusion We next provide empirical evidence that the speed of diffusion of each infectious disease is shaped by international trade (see also Online Appendix G.1). We

\footnotetext{
${ }^{4}$ For a historical discussion of the relationship between globalization and disease diffusion, see Kenny (2021).

${ }^{5}$ See for example Diamond (1998) and Nunn and Qian (2010). We focus on the relationship between globalization and the speed of diffusion of infectious diseases rather than mortality, because mortality can be heavily influenced by public health improvements, as discussed for example in Chapters 6 and 7 of Kenny (2021).

${ }^{6}$ Traditionally, it was thought that this bacterium was mainly spread by rat fleas. More recent research suggests that it also can be spread by human and cat fleas, or through infected meat, or oral transmission between humans.

${ }^{7}$ For the case of Covid-19, in Online Appendix G.1.4, we also provide evidence on the intensive margin growth of infections (and how it is shaped by infections in other countries) once the first infection has occurred.

${ }^{8}$ These findings are consistent with the epidemiological literature that argues that global transport networks have accelerated disease diffusion, as reviewed in Tatem et al. (2006), and discussed in Online Appendix G.2. Although, of course, one also needs to account for differences in infection rates across diseases.
} 
Figure 1: Arrival Times for the Plague, 1957-8 Influenza and Covid-19
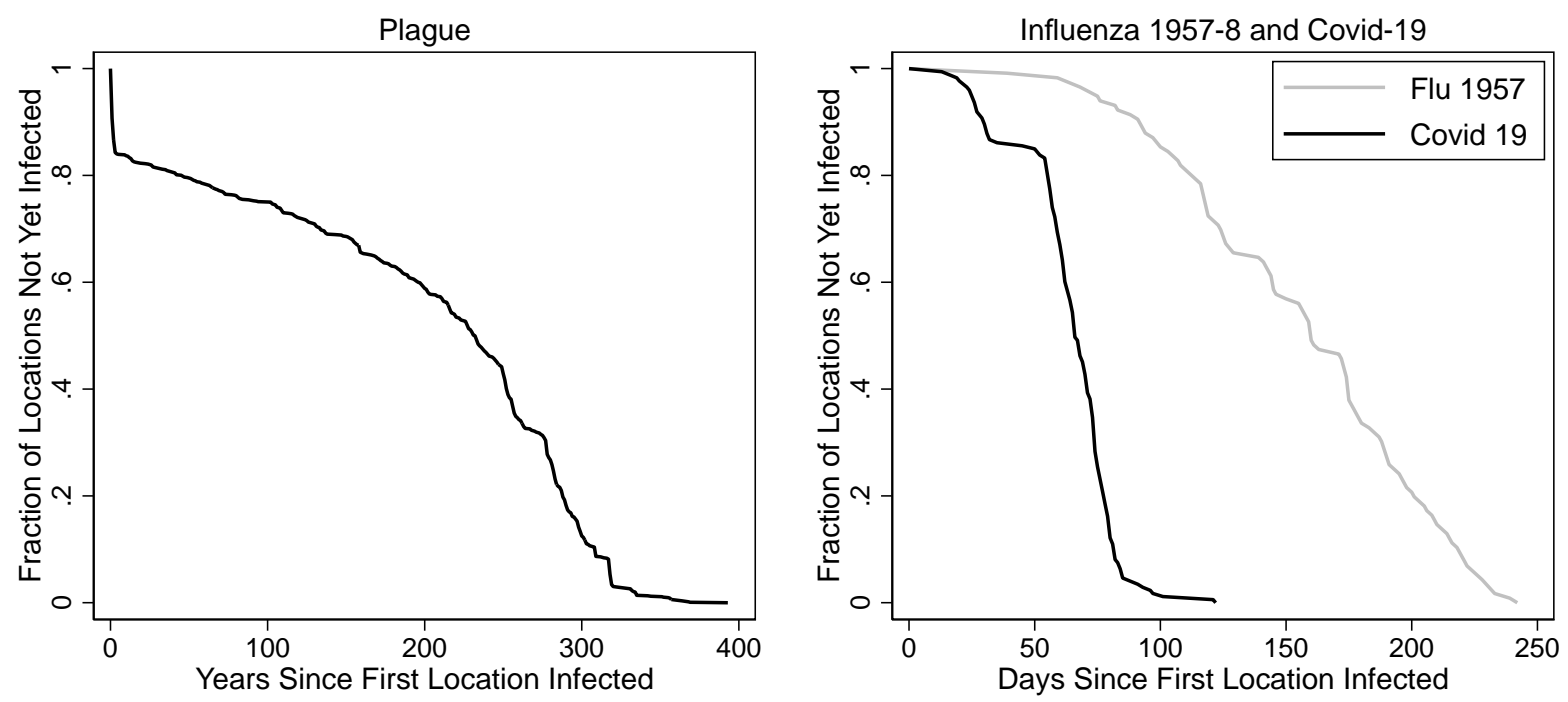

Note: Arrival time defined as the difference in time between the first outbreak of a disease in a location and its first outbreak anywhere; left panel shows arrival times in years for the plague across European cities from 1347-1760; right panel shows arrival times in days for 1957-8 influenza and Covid-19 across countries.

focus on the initial diffusion of each disease, and use measures of international trade from before the outbreak of each disease, in order to abstract from public policy interventions and behavioral responses.

In our baseline specification, we regress the arrival time of each disease on these pre-existing trade measures. Much of the variation in these pre-existing trade measures is driven by bilateral geographical distance. But one potential concern is that there could be omitted variables that affect disease diffusion and are correlated with geographical distance (e.g., movements of animals that can act as reservoirs for the disease). To address this concern, we also report specifications, in which we control separately for geographical distance. In each specification, we standardize variables to have a mean of zero and a standard deviation of one, such that the estimated coefficients correspond to $\beta$-coefficients. In Column (1) of Table 1, we report the estimation results for the plague. Since data on bilateral trade between European cities during the medieval period are unavailable, we follow the existing historical literature on the plague in measuring trade access using the inverse of distance from the nearest Old World trade route. Even after controlling for log geographical distance from the first European plague outbreak in Messina, we find a negative and statistically significant coefficient on log trade access, implying that the plague diffused faster to cities with better trade access.

In Column (2) of Table 1, we report the results for the 1957-8 influenza, where there are only 52 countries for which we observe both influenza arrival times and positive bilateral trade (exports plus imports in 1956) with China, as the country with the first outbreak. Despite the relatively small sample, we find a negative and statistically significant coefficient on log trade with China, 
implying shorter arrival times for the influenza for countries that trade more intensively with China, even after controlling for log geographical distance. ${ }^{9}$

Table 1: Arrival Times for Plague, Influenza 1957-8 and Covid-19

\begin{tabular}{|c|c|c|c|c|c|}
\hline & $\begin{array}{c}(1) \\
\text { Log } \\
\text { Arrival }\end{array}$ & $\begin{array}{c}(2) \\
\text { Log } \\
\text { Arrival }\end{array}$ & $\begin{array}{c}(3) \\
\text { Log } \\
\text { Arrival }\end{array}$ & $\begin{array}{c}(4) \\
\text { Log } \\
\text { Arrival }\end{array}$ & $\begin{array}{c}(5) \\
\text { Log } \\
\text { Arrival }\end{array}$ \\
\hline & Plague & Influenza 1957-8 & Covid-19 & Covid-19 & Covid-19 \\
\hline Log Trade Access & $\begin{array}{c}-0.218^{* * *} \\
(0.0349)\end{array}$ & & & & \\
\hline Log Trade & & $\begin{array}{l}-0.176^{*} \\
(0.1048)\end{array}$ & $\begin{array}{r}-0.525^{* * *} \\
(0.0742)\end{array}$ & $\begin{array}{r}-0.238^{* * *} \\
(0.0804)\end{array}$ & $\begin{array}{c}0.0195 \\
(0.1012)\end{array}$ \\
\hline Log Distance & $\begin{array}{l}0.108^{* * *} \\
(0.0302)\end{array}$ & $\begin{array}{l}0.560^{* * *} \\
(0.0867)\end{array}$ & $\begin{array}{c}0.301^{* * *} \\
(0.0819)\end{array}$ & $\begin{array}{c}0.227^{* * *} \\
(0.0819)\end{array}$ & $\begin{array}{c}0.0665 \\
(0.0897)\end{array}$ \\
\hline Log Migrant Stock & & & & $\begin{array}{c}-0.440^{* * *} \\
(0.0920)\end{array}$ & $\begin{array}{r}-0.279^{* * *} \\
(0.0945)\end{array}$ \\
\hline Log Arrivals-Departures & & & & & $\begin{array}{c}-0.512^{* * *} \\
(0.1259)\end{array}$ \\
\hline Observations & 1,149 & 52 & 172 & 152 & 149 \\
\hline R-squared & 0.072 & 0.439 & 0.462 & 0.538 & 0.576 \\
\hline
\end{tabular}

Note: Observations in Column (1) are European cities; Observations in Columns (2)-(5) are countries; All variables are standardized to have a mean of zero and a standard deviation of one; Arrival is the difference in time between the first outbreak of a disease in a location and the first outbreak anywhere; Trade Access is the inverse of the shortest distance to an Old World Trade Route; Trade is the sum of the value of each country's exports and imports with China (the country with the first outbreak of Influenza 1957-8 and Covid-19); trade is measured in the year before each of these pandemics (1956 and 2019, respectively); Distance is geographical distance from the location of the first outbreak; Migrant stock is the total number of immigrants in a country from China plus the total number of ex-patriots in China from that country; Arrivals-Departures is the total number of people arriving in a country from China plus the total number of people arriving in China from that country (including migrants, business travellers and tourists); heteroskedasticity robust standard errors in parentheses; ${ }^{* * *}$ denotes significance at the 1 percent level; ** denotes significance at the 5 percent level; * denotes significance at the 10 percent level.

In Column (3) of Table 1, we show that we find the same pattern of results for Covid-19, with a negative and statistically significant coefficient on log trade with China (in 2019), even after controlling for log geographical distance, where again China is the country with the first outbreak. ${ }^{10}$ The natural mechanism through which trade affects disease diffusion is international travel. For Covid-19, we can provide further evidence on this mechanism, by including as controls other measures of international linkages between countries for which data are available.

In Column (4), we include the log stock of migrants in China from each country (including both immigrants and ex-patriots). Consistent with the idea that disease can be transmitted through

\footnotetext{
${ }^{9}$ If we include zero trade flows by estimating the equation in levels rather than logs, we have 117 countries for which we observe arrival times, and find an estimated coefficient (standard error) of -0.135 (0.0145). We find similar results for the 1968-9 influenza, with negative and statistically significant trade coefficients in both levels and logs.

${ }^{10}$ These results are again consistent with a large historical literature that emphasizes the role of international travel and trade in disease diffusion, as discussed in Fauci (2005) and Online Appendix G.2.
} 
people movements associated with both migration and trade, we find negative and statistically significant coefficients for both variables. In Column (5), we further augment this specification with the log total number of arrivals and departures between each country and China (including migration, business travel, and tourism). Consistent with trade affecting disease diffusion through travel, we find that the estimated trade coefficient is no longer significant once we control for total arrivals and departures. We continue to find a negative and statistically significant coefficient on the log migrant stock, which could reflect measurement error in total arrivals and departures, such that the migration stock is proxying for unobserved people flows associated with migration. ${ }^{11}$

In sum, for all three infectious diseases, we find that the speed of diffusion of the disease is systematically related to international trade, even after controlling for distance. Additionally, for Covid-19, we find that this effect of international trade operates through movements of people, which is consistent with the scientific mechanism through which Covid-19 is transmitted (which requires exposure to infectious respiratory fluids through face-to-face interactions). While we focus here in the main text on the extensive margin of disease diffusion, in Online Appendix G.1.4 we use our additional data for Covid-19 to provide evidence on the intensive margin of the rate of growth of infections once the first infection has occurred. We show that countries that trade more with partners with high relative levels of infections experience more rapid rates of growth of infection, even after controlling for the time since their own first infection.

International Travel and Trade We now provide further evidence on the relationship between international travel and trade using direct data on bilateral travel, trade and tariffs (see also Online Appendix H.1). ${ }^{12}$

We measure international travel using data on the bilateral number of air passengers from the Origin and Destination (OFOD) Database of the International Civil Aviation Organization (ICAO) from 1982-2019. We begin by examining the correlation between international travel and trade. We regress the log of bilateral air passengers on the log of bilateral trade (exports plus imports), including origin-destination and year fixed effects. The inclusion of the origin-destination fixed effects implies that the estimated coefficient is identified from the relationship between changes in international travel and changes in international trade over time. As reported in Column (1) of Table 2, we find a positive and statistically significant correlation between international travel and trade, with a one percent increase in the value of bilateral trade associated with a 0.217 percent increase in bilateral air passengers.

We find that this pattern of results is robust across a range of different specifications. One potential concern is that air passengers could underestimate bilateral travel, because it abstracts from travel by land or sea. In Column (2), we find similar results if we restrict attention to origindestination pairs more than 3,000 kilometers apart, for which air travel is likely to be the dominant

\footnotetext{
${ }^{11}$ As a further specification check, we report placebo specifications in Online Appendix G.1 in which we show that a range of measures of international financial linkages are statistically insignificant once we control for total arrivals and departures, consistent with the travel-based mechanism in the model.

${ }^{12}$ For a review of the existing empirical literature on the role played by business travel in international trade, see Online Appendix H.2.
} 
Table 2: International Travel and Trade

\begin{tabular}{lccccc}
\hline \hline & $\begin{array}{c}(1) \\
\text { Log Air } \\
\text { Passengers }\end{array}$ & $\begin{array}{c}(2) \\
\text { Log Air } \\
\text { Passengers }\end{array}$ & $\begin{array}{c}(3) \\
\text { Log Air } \\
\text { Passengers }\end{array}$ & $\begin{array}{c}(4) \\
\text { Log Air } \\
\text { Passengers }\end{array}$ & $\begin{array}{c}(5) \\
\text { Log Air } \\
\text { Passengers }\end{array}$ \\
\hline Log Trade & $0.217^{* * *}$ & $0.293^{* * *}$ & & $0.399^{* *}$ & \\
& $(0.0111)$ & $(0.0173)$ & & $(0.1574)$ & \\
Log Trade Land/Sea & & & & & \\
& & & $0.230^{* * *}$ & & \\
Log Tariff & & & & & \\
& & & & & $-1.0134)$ \\
Estimation & OLS & OLS & OLS & IV & OLS \\
Sample & All & $>3,000 \mathrm{~km}$ & All & All & All \\
Origin-Destination FEs & Yes & Yes & Yes & Yes & Yes \\
Year FEs & Yes & Yes & Yes & Yes & Yes \\
Observations & 124,597 & 65,332 & 96,197 & 68,734 & 71,376 \\
R-squared & 0.763 & 0.771 & 0.781 & - & 0.790 \\
First-stage F-statistic & - & - & - & 100.8 & - \\
\hline \hline
\end{tabular}

Note: Panel of origin-destination-year observations; Air passengers is total number of airline passengers from an origin to a destination in a given year; Trade is the average of exports and imports for each origin and destination in a given year; Trade Land/Sea is the average of exports and imports for Harmonized System (HS) 6-digit products for which air transport accounts for less than 20 percent of the value of trade; Log tariff is the average of the $a d$ valorem bilateral tariff in the origin and destination countries; Origin-Destination FEs are origin-destination fixed effects; Year FEs are year fixed effects; Sample in Column (2) is restricted to origin-destination pairs with bilateral distances greater than 3,000 km; In Column (4), Log trade is instrumented with log tariff; First-stage F-statistic is the F-statistic for the statistical significance of the instrument in the first-stage regression; the R-squared for the second-stage regression is not reported in the IV specification, because it does not have a meaningful interpretation; Standard errors in parentheses are clustered by origin-destination pair; ${ }^{* * *}$ denotes significance at the 1 percent level; ** denotes significance at the 5 percent level; * denotes significance at the 10 percent level.

mode of transport. Another potential concern is that aircrafts are used to transport both people and goods, which could introduce a mechanical correlation between air travel and trade. In Column (3), we find similar results if we restrict attention to trade in goods for which air transport accounts for less than 20 percent of the value of trade, confirming that our findings are not driven by such a mechanical correlation. ${ }^{13}$

Our theoretical model below suggests that the estimated positive correlation between travel and trade in Columns (1)-(3) need not have a causal interpretation, because both variables are jointly determined by trade and travel frictions. To explore further the role of trade frictions, we next consider a direct measure of trade policy barriers in the form of tariffs. In Column (4), we report instrumental variables (IV) estimates of our baseline specification from Column (1), in which we instrument bilateral trade using bilateral tariffs. We find a positive and statistically significant relationship between international travel and trade predicted by tariffs. In the corresponding firststage regression, we find that bilateral tariffs are a powerful predictor of bilateral trade, with a

\footnotetext{
${ }^{13}$ COMTRADE data are not reported by origin-destination-product-mode of shipment. Therefore, we combine trade data by origin-destination-product with separate information on the importance of air trade for each product.
} 
first-stage F-statistic well above the conventional threshold of 10.

The exclusion restriction for the IV specification in Column (4) is that bilateral tariffs only affect bilateral travel through bilateral trade. This exclusion restriction could be violated if there are other channels through which bilateral tariffs affect bilateral travel (e.g., through the travel of trade negotiators). In Column (5), we report the corresponding reduced-form regression of bilateral travel on bilateral tariffs, which captures all channels through which bilateral travel and tariffs are related. We find a negative and statistically significant coefficient, implying that trade liberalization is correlated with increased bilateral travel. Nevertheless, this correlation is again not necessarily causal, because bilateral tariffs are the result of a political economy process, which itself could be endogenous to bilateral travel between countries.

Although it is challenging to ever fully address this endogeneity concern, a growing empirical literature has used quasi-experimental variation to provide evidence in support of a causal relationship between bilateral trade and travel, as discussed further in Online Appendix H.2. For example, Söderlund (2020) uses the liberalization of airspace in the Soviet Union in the mid-1980s, and shows that the resulting reductions in the costs of business travel led to increased trade, even for goods not typically transported by air. Additionally, Campante and Yanagizawa-Drott (2018) uses the technological limitations of air travel beyond 6,000 miles, and shows that city-pairs just below this distance threshold are substantially more likely to have direct flight connections. Locations close to airports with a larger share of links below this 6,000 mile threshold are found to experience increased business linkages and measures of local economic activity.

Motivated by these empirical findings on disease diffusion, international trade and international travel, we now turn to develop our baseline theoretical framework. We discuss additional empirical evidence relating to the novel predictions of our theoretical framework below.

\section{Baseline Economic Model}

In this section we develop a stylized model of the global economy in which international trade is sustained by face-to-face interactions. Our main goal is to develop the simplest model in which both international trade and face-to-face interactions are jointly determined in general equilibrium. With that in mind, we focus on a baseline static one-sector, two-country world in which individuals use labor to produce differentiated goods that are exchanged exclusively via face-to-face interactions in competitive markets. Although this specification is intentionally stylized, we end this section by discussing briefly how our model can be generalized to a wide range of settings while preserving its main insights. We develop these extensions more fully in Online Appendix C.

\subsection{Environment}

Consider a world with two locations: East and West, indexed by $i$ or $j$. We denote by $\mathcal{J}$ the set of countries in the world, so $\mathcal{J}=\{$ East,West $\}$. Location $i \in \mathcal{J}$ is inhabited by a continuum of measure $L_{i}$ of households, and each household is endowed with the ability to produce a differentiated 
variety using labor as the only input in production. We denote by $w_{i}$ the wage rate in country $i$. We abstract from intertemporal borrowing and lending and hence income equals expenditure.

Trade is costly. There are iceberg bilateral trade cost $\tau_{i j}=t_{i j}\left(d_{i j}\right)^{\delta}$, when shipping from $j$ back to $i$, where $d_{i j} \geq 1$ is the symmetric distance between $i$ and $j$, and $t_{i j}$ is a man-made additional trade friction imposed by $i$ on imports from country $j$. We let these man-made trade costs be potentially asymmetric reflecting the fact that one country may impose higher restrictions to trade (e.g., tariffs, or delays in goods clearing customs) than the other country. For simplicity, there are no man-made frictions to internal shipments, so $t_{i i}=1$ and $\tau_{i i}=\left(d_{i i}\right)^{\delta}$, where $d_{i i}<d_{i j}$ for $j \neq i$ can be interpreted as the average internal distance in country $i=$ East, West.

Each of the $L_{i}$ households in country $i$ is formed by two individuals. One of these individuals - the seller - is in charge of producing and selling the household-specific differentiated variety from their home, while the other individual - the buyer - is in charge of procuring varieties for consumption from other households in each of the two locations. We let all households in country $i$ be equally productive in manufacturing varieties, with one unit of labor delivering $Z_{i}$ units of goods. Goods markets are competitive and sellers make their goods available at marginal cost.

Households have CES preferences over differentiated varieties, with an elasticity of substitution $\sigma>1$ regardless of the origin of these varieties, and they derive disutility from the buyer spending time away from home. More specifically, a household in country $i$ incurs a utility cost

$$
c_{i j}\left(n_{i j}\right)=\frac{c}{\phi} \mu_{i j}\left(d_{i j}\right)^{\rho}\left(n_{i j}\right)^{\phi},
$$

whenever the household's buyer secures $n_{i j}$ varieties from location $j$, at a distance $d_{i j} \geq 1$ from $i$. The parameter $\mu_{i j}$ captures travel restrictions imposed by country $j$ 's government on visitors from $i$. The parameter $c$ governs the cost of travel and we assume it is large enough to ensure an interior solution in which $n_{i j} \leq L_{j}$ for all $i$ and $j \in \mathcal{J}$. We assume that whenever $n_{i j}<L_{j}$, the set of varieties procured from $j$ are chosen at random, so if all households from $i$ procure $n_{i j}$ from $j$, each household's variety in $j$ will be consumed by a fraction $n_{i j} / L_{j}$ of households from $i$.

Welfare of households in location $i$ is then given by

$$
W_{i}=\max _{n_{i i}, n_{i j}, q_{i i}(\cdot), q_{i j}(\cdot)}\left[\left(\sum_{j \in \mathcal{J}} \int_{0}^{n_{i j}} q_{i j}(k)^{\frac{\sigma-1}{\sigma}} d k\right)^{\frac{\sigma}{\sigma-1}}-\frac{c}{\phi} \sum_{j \in \mathcal{J}} \mu_{i j}\left(d_{i j}\right)^{\rho}\left(n_{i j}\right)^{\phi}\right],
$$

subject to the household's budget constraint

$$
\sum_{j \in \mathcal{J}} \int_{0}^{n_{i j}} p_{i j}(k) q_{i j}(k) d k=w_{i}
$$

where $p_{i j}(k)$ and $q_{i j}(k)$ denote the price and quantity consumed in $i$ of the variety produced in $j$ by household $k$, and $w_{i}$ is household income. 


\subsection{Equilibrium}

Let us first consider the consumption choices of a representative household in country $i$ for a given $n_{i j}$. The problem in (2), yields

$$
q_{i j}=\frac{w_{i}}{\left(P_{i}\right)^{1-\sigma}}\left(\frac{\tau_{i j} w_{j}}{Z_{j}}\right)^{-\sigma}
$$

where $w_{j} / Z_{j}$ is the common free-on-board price of all varieties produced in location $j, \tau_{i j}$ are trade costs when shipping from $j$ to $i$, and $P_{i}$ is a price index given by

$$
P_{i}=\left(\sum_{j \in \mathcal{J}} n_{i j}\left(\frac{\tau_{i j} w_{j}}{Z_{j}}\right)^{1-\sigma}\right)^{1 /(1-\sigma)}
$$

In order to characterize each household's choice of $n_{i j}$, we first plug equations (4) and (5) into equation (2) to obtain

$$
W_{i}=\max _{n_{i i}, n_{i j}} w_{i}\left[\left(\sum_{j \in \mathcal{J}} n_{i j}\left(\frac{\tau_{i j} w_{j}}{Z_{j}}\right)^{1-\sigma}\right)^{\frac{1}{(\sigma-1)}}-\frac{c}{\phi} \sum_{j \in \mathcal{J}} \mu_{i j}\left(d_{i j}\right)^{\rho}\left(n_{i j}\right)^{\phi}\right] .
$$

From the first-order condition for $n_{i j}$, we obtain the following gravity equation for face-to-face interactions that is log-separable in origin and destination terms and bilateral frictions:

$$
n_{i j}=\left(c(\sigma-1) \mu_{i j}\right)^{-1 /(\phi-1)}\left(d_{i j}\right)^{-\frac{\rho+(\sigma-1) \delta}{\phi-1}}\left(\frac{t_{i j} w_{j}}{Z_{j} P_{i}}\right)^{-\frac{\sigma-1}{(\phi-1)}}\left(\frac{w_{i}}{P_{i}}\right)^{1 /(\phi-1)} .
$$

Evidently, natural and man-made barriers to trade $\left(d_{i j}, t_{i j}\right)$ and to travel $\left(\mu_{i j}\right)$ tend to reduce the number of interactions sought by agents from country $i$ in country $j$. As we show in Online Appendix B.1, for the second-order conditions to be satisfied for all values of $\mu_{i j}, d_{i j}$, and $t_{i j}$, we require $\phi>1 /(\sigma-1)$ and $\sigma>2$. Bilateral imports satisfy an analogous gravity equation:

$$
X_{i j}=n_{i j} p_{i j} q_{i j} L_{i}=\left(c(\sigma-1) \mu_{i j}\right)^{-\frac{1}{\phi-1}}\left(d_{i j}\right)^{-\frac{\rho+\phi(\sigma-1) \delta}{\phi-1}}\left(\frac{t_{i j} w_{j}}{Z_{j} P_{i}}\right)^{-\frac{\phi(\sigma-1)}{\phi-1}}\left(\frac{w_{i}}{P_{i}}\right)^{\frac{1}{\phi-1}} w_{i} L_{i} .
$$

Therefore trade shares can be written as the following constant elasticity import demand system,

$$
\pi_{i j}=\frac{X_{i j}}{\sum_{\ell \in \mathcal{J}} X_{i \ell}}=\frac{\left(\Upsilon_{i j}\right)^{-\varepsilon}\left(w_{j} / Z_{j}\right)^{-\frac{\phi(\sigma-1)}{\phi-1}}}{\sum_{\ell \in \mathcal{J}}\left(\Upsilon_{i \ell}\right)^{-\varepsilon}\left(w_{\ell} / Z_{\ell}\right)^{-\frac{\phi(\sigma-1)}{\phi-1}}}
$$

where we have defined the composite trade friction as

$$
\left(\Upsilon_{i j}\right)^{-\varepsilon} \equiv\left(\mu_{i j}\right)^{-\frac{1}{\phi-1}}\left(d_{i j}\right)^{-\frac{\rho+\phi(\sigma-1) \delta}{\phi-1}}\left(t_{i j}\right)^{-\frac{\phi(\sigma-1)}{\phi-1}}
$$

which encompasses travel frictions $\left(\mu_{i j}\right)$, transport costs $\left(d_{i j}\right)$, and trade frictions $\left(t_{i j}\right)$. Note that the distance elasticity is affected by the standard substitutability $\sigma$, but also by the traveling cost 
elasticity $\rho$, and by the convexity $\phi$ of the traveling costs. It is clear that both $\rho>0$ and $\phi>1$ increase the distance elasticity relative to a standard Armington model (in which the distance elasticity would be given by $\delta(\sigma-1)) .{ }^{14}$

Finally, from the equality between country $i$ 's income and expenditure on the goods that it produces, we obtain the following system of equations that determines equilibrium wages:

$$
\pi_{i i}\left(w_{i}, w_{j}\right) w_{i} L_{i}+\pi_{j i}\left(w_{i}, w_{j}\right) w_{j} L_{j}=w_{i} L_{i}
$$

where $\pi_{i i}\left(w_{i}, w_{j}\right)$ and $\pi_{j i}\left(w_{i}, w_{j}\right)$ are given by equation (9) and we choose the wage in one country as the numeraire. This general-equilibrium condition (11) takes the same form as in standard gravity models in Alvarez and Lucas (2007), Allen and Arkolakis (2014), or Allen et al. (2020). Therefore, there exists a unique vector of equilibrium wages $\mathbf{w}^{*}=\left(w_{i}, w_{j}\right) \in \mathbb{R}_{++}^{2}$, as summarized in Proposition B.1 in Online Appendix B.3.

We obtain intuitive comparative statics for the impact of international trade or travel frictions $\left(d_{i j}, t_{i j}, t_{j i}, \mu_{i j}, \mu_{j i}\right)$ on bilateral interactions. Combining equations (4), (8), and (9), we can express bilateral interactions as:

$$
n_{i j}(\mathbf{w})=\left(\frac{t_{i j}\left(d_{i j}\right)^{\delta} w_{j}}{P_{i}(\mathbf{w}) Z_{j}}\right)^{\sigma-1} \pi_{i j}(\mathbf{w}),
$$

where $\pi_{i j}(\mathbf{w})$ is given in (9) and $P_{i}(\mathbf{w})$ in equation (B.1) in Online Appendix B.2. A decline in any international trade or travel friction leads to: (a) a decline in the rates $\left(n_{i i}\right.$ and $\left.n_{j j}\right)$ at which individuals will meet individuals in their own country; and (b) an increase in the rates at which individuals will meet individuals from the other country $\left(n_{i j}\right.$ and $\left.n_{j i}\right)$ (see Proposition B.2 in Online Appendix B.4). Therefore, the more open are economies to flows of goods and people, the larger will be international interactions and the lower will be domestic interactions.

In contrast, the effect of international trade and travel frictions on the overall number of interactions is more subtle and in general ambiguous, because domestic and foreign interactions are imperfect substitutes for one another. In Proposition B.3 in Online Appendix B.5, we show that if countries are symmetric $\left(L_{i}=L, Z_{i}=Z\right.$, and $\Upsilon_{i j}=\Upsilon$ for all $i$ ), a decline in any (symmetric) international friction leads to an overall increase in human interactions $\left(n_{i i}+n_{i j}\right)$ experienced by both household buyers and sellers. We use these comparative statics in Section 4 below, when we study the relationship between disease diffusion and globalization

We have intentionally developed a stylized model of international trade and face-to-face interactions, which allows us to derive predictions for the relationship between globalization and pandemics as transparently and sharply as possible. In Online Appendix C, we show that the main predictions of our theoretical framework are not sensitive to these stylized features and continue to hold under a wide range of generalizations. For instance, we work out an extension in which households have access to two alternative technologies for procuring consumption goods, one involving

\footnotetext{
${ }^{14} \mathrm{An}$ implication of this constant elasticity gravity equation representation is that our model falls within the class of models discussed in Arkolakis et al. (2012), in which the domestic trade share is a sufficient statistic for welfare, as shown in Online Appendix B.2.
} 
travel, and the other one involving remote interactions. We also develop further generalizations of this specification, in which the shares of varieties sourced differ across households, and potentially feature full segmentation between 'jet setters' and 'home bodies,' as well as a dynamic version of our framework in which face-to-face interactions are only necessary to initiate a commercial link between a buyer and a seller. In that same Online Appendix C, we discuss other extensions involving multiple sectors with varying degrees of face-to-face interactions, multiple countries, an alternative specification of travel costs in terms of labor rather than utility, intermediate-input trade, scale economies, and alternative market structures. Across each of these different extensions and generalizations, we show that the insights from our baseline specification continue to hold.

\section{Trade, Travel and Disease Diffusion}

We now embed our gravity model of trade and travel into a model of disease dynamics. We interpret our model as describing a standard "day" in the household. In the morning the buyer in each household in $i$ leaves the house and visits $n_{i i}$ sellers in $i$ and $n_{i j}$ sellers in $j$, procuring goods from each of those households. For simplicity, we assume that buyers do not travel together or otherwise meet each other. While the buyer visits other households and procures goods, the seller in each household sells its own goods to visitors to their household. There will be $n_{i i}$ domestic visitors and $n_{j i}$ foreign visitors. In the evening, the two members of the household reunite.

\subsection{Preliminaries}

With this background in mind, consider now the dynamics of contagion. As in the standard epidemiological model, we divide the population at each point in time into Susceptible households, Infectious households, and Recovered households (we will incorporate deaths in the next section). We think of the health status as being a household characteristic, implicitly assuming a perfect rate of transmission within the household (they enjoy a passionate marriage), and also that recovery is experienced simultaneously by all household members. For simplicity, we ignore the possibility that a vaccine puts an end to an epidemic before herd immunity is achieved.

Throughout this section, we focus on the role of trade and travel in shaping disease dynamics through the intensity of domestic and foreign interactions. In particular, we abstract from any impact of the disease on the ability to work and trade, or on mortality. Therefore, there are no general equilibrium effects of the pandemic on labor supply, and there is also no incentive for agents to change their individual behavior. As a result, domestic and foreign interactions are time-invariant throughout the course of the pandemic.

Even with these simplifications, the endogeneity of domestic and foreign interactions to trade and travel costs implies that the gravity model of trade and the SIR model of disease dynamics interact in subtle ways. First, we establish an epidemiological externality between countries, such that the condition for a pandemic to occur depends on domestic interactions in the most unhealthy country, where the intensity of these domestic interactions differs between the open and closed 
economies. Second, the constant elasticity gravity equation implies that domestic and foreign interactions are substitutes, in the sense that a reduction in trade costs increases foreign interactions relative to domestic ones. Therefore, reductions in trade costs can either increase or decrease the range of parameters where a pandemic occurs, and the severity of the pandemic when it does occur, depending on the relative levels of infections at home and abroad.

In Section 5, we generalize the analysis to allow the disease to affect the ability to work and trade, as well as mortality. But we assume that agents are unaware of the source of the infection, and hence have no incentive to change their individual behavior. In this case, there is a further interaction between trade and disease dynamics, because changes in relative labor supplies give rise to a form of general equilibrium social distancing. In Section 6, we further enrich the analysis to allow agents to become aware of the source of the infection, and to adjust the intensity of their interactions based on the threat of infection. This introduces yet another interaction between trade and disease dynamics, because of individual-level social distancing in response to the threat of infection.

\subsection{The Dynamic System}

The share of households of each type in country $i$ evolves according to the following laws of motion (we ignore time subscripts for now to keep the notation tidy):

$$
\begin{aligned}
\dot{S}_{i} & =-2 n_{i i} \alpha_{i} S_{i} I_{i}-n_{i j} \alpha_{j} S_{i} I_{j}-n_{j i} s \alpha_{i} S_{i} I_{j} \\
\dot{I}_{i} & =2 n_{i i} \alpha_{i} S_{i} I_{i}+n_{i j} \alpha_{j} S_{i} I_{j}+n_{j i} \alpha_{i} S_{i} I_{j}-\gamma_{i} I_{i} \\
\dot{R}_{i} & =\gamma_{i} I_{i}
\end{aligned}
$$

To better understand this system, focus first on how infections grow in equation (14). The first term $2 n_{i i} \alpha_{i} S_{i} I_{i}$ in this equation captures newly infected households in country $i$. Sellers in $i$ receive (in expectation) $n_{i i}$ domestic buyers, while buyers meet up with $n_{i i}$ domestic sellers. The household thus jointly has $2 n_{i i}$ domestic contacts. In those encounters, a new infection occurs with probability $\alpha_{i}$ whenever one of the agents is susceptible (which occurs with probability $S_{i}$ ) and the other agent is infectious (which occurs with probability $\left.I_{i}\right) .{ }^{15}$ The second term of equation (14) reflects new infections of country $i$ 's households that occur in the foreign country when susceptible buyers from $i$ (of which there are $S_{i}$ ) visit foreign households with infectious sellers. There are $n_{i j}$ of those meetings, leading to a new infection with probability $\alpha_{j}$ whenever the foreign seller is infectious (which occurs with probability $I_{j}$ ). Finally, the third term in (14) reflects new infections associated with susceptible sellers in country $i$ receiving infectious buyers from abroad (country $j$ ). Each susceptible domestic buyer (constituting a share $S_{i}$ of $i$ 's population) has $n_{j i}$ such meetings, which cause an infection with probability $\alpha_{i}$ whenever the foreign buyer is infectious (which occurs

\footnotetext{
${ }^{15}$ In summing the buyer and seller domestic contact rates to obtain a domestic contact rate of $2 n_{i i}$ for the household, we use the continuous time property that there is zero probability that the buyer and seller are simultaneously infected at exactly the same instant.
} 
with probability $I_{j}$ ). The final term in equation (14) simply captures the rate at which infectious individuals recover $\left(\gamma_{i}\right)$, and note that we assume that this recovery rate only depends on the country in which infected agents reside, regardless of where they got infected.

Once the equation determining the dynamics of new infections is determined, the one determining the change of susceptible agents in (13) is straightforward to understand, as it just reflects a decline in the susceptible population commensurate with new infections. Finally, equation (15) governs the transition from infectious households to recovered households.

We allow the epidemiological parameters $\left(\alpha_{i}, \gamma_{i}\right)$ to differ across countries, which is consistent with the very different experiences of countries during the Covid-19 pandemic. One source for these differences is culture. For example, Japan was relatively less affected by Covid-19, because of its culture of mask wearing in public. Another source is differences in public health technology, including administrative capability and medical resources. For example, South Korea was relatively less affected by Covid-19, because of an effective contact tracing scheme. In contrast, India was relatively severely affected, because of a lack of hospital capacity and available treatments. ${ }^{16}$

\subsection{Open-Economy Pandemic}

In the closed-economy $\left(n_{i j}=n_{j i}=0\right)$, our model reduces to a standard SIR model following Kermack and McKendrick $(1927,1932)$, in which $\beta_{i}=2 n_{i i}$ is the so-called contact rate. If $\mathcal{R}_{0 i}=$ $\beta_{i} / \gamma_{i}<1$, an epidemic-free equilibrium is globally stable. In contrast, if $\mathcal{R}_{0 i}=\beta_{i} / \gamma_{i}>1$, a small initial level of infections necessarily induces an epidemic to occur.

In the open economy, the system of equations (13)-(15) shares properties with multi-group SIR models, if bilateral interactions $\left(n_{i j}\right)$ are taken as given. However, a key difference from these existing multi-group SIR models is that bilateral interactions are endogenously determined by international trade and travel frictions in our framework. This will allow us to study, in the next two subsections, how the emergence and severity of pandemics is shaped by the level of trade integration in the world economy.

In parallel to the closed economy case, we can define $\mathcal{R}_{0}$ in the two-country system as the expected number of secondary cases produced by a single (typical) infection, starting from the pandemic-free equilibrium in which $S_{i}=S_{j} \simeq 1$ and $I_{i}=I_{j} \simeq 0$. Building on existing results for multi-group SIR models, we can compute $\mathcal{R}_{0}$ by writing the system of equations determining the dynamics of infections in each country as follows: ${ }^{17}$

$$
\left[\begin{array}{c}
\dot{I}_{i} \\
\dot{I}_{j}
\end{array}\right]=\underbrace{\left[\begin{array}{cc}
2 \alpha_{i} n_{i i} S_{i} & \left(\alpha_{j} n_{i j}+\alpha_{i} n_{j i}\right) S_{i} \\
\left(\alpha_{j} n_{i j}+\alpha_{i} n_{j i}\right) S_{j} & 2 \alpha_{j} n_{j j} S_{j}
\end{array}\right]}_{F}\left[\begin{array}{c}
I_{i} \\
I_{j}
\end{array}\right]-\underbrace{\left[\begin{array}{cc}
\gamma_{i} & 0 \\
0 & \gamma_{j}
\end{array}\right]}_{V}\left[\begin{array}{c}
I_{i} \\
I_{j}
\end{array}\right] .
$$

\footnotetext{
${ }^{16}$ See Kang et al. (2021) on South Korea's contact tracing and the New York Times article "The Night the Oxygen Ran Out" (June 28, 2021) for an account of India's problems with hospital capacity.

${ }^{17}$ In particular, we build on the analysis of multi-group SIR models in Hethcote (1978), Hethcote and Thieme (1985), Diekmann et al. (1990), van den Driessche and Watmough (2002), and Magal et al. (2016).
} 
The dynamics of infections in this system depend on the properties so-called next generation matrix $F V^{-1}$ (evaluated at $t=t_{0}$, for which $S_{i}\left(t_{0}\right)=S_{j}\left(t_{0}\right) \simeq 1$ ). In particular, $\mathcal{R}_{0}$ is given by the spectral radius $\left(\rho\left(F V^{-1}\right)\right)$ of this next generation matrix:

$$
\mathcal{R}_{0}=\frac{1}{2}\left(\frac{2 \alpha_{i} n_{i i}}{\gamma_{i}}+\frac{2 \alpha_{j} n_{j j}}{\gamma_{j}}\right)+\frac{1}{2} \sqrt{\left(\frac{2 \alpha_{i} n_{i i}}{\gamma_{i}}-\frac{2 \alpha_{j} n_{j j}}{\gamma_{j}}\right)^{2}+4 \frac{\left(\alpha_{j} n_{i j}+\alpha_{i} n_{j i}\right)^{2}}{\gamma_{i} \gamma_{j}}}
$$

We thus obtain the following characterization of the open economy equilibrium.

Proposition 1 Assume that there is trade between the two countries (i.e., $\alpha_{j} n_{i j}+\alpha_{i} n_{j i}>0$ ). If $\mathcal{R}_{0} \leq 1$, the no-pandemic equilibrium is the unique stable equilibrium. If $\mathcal{R}_{0}>1$, the no-pandemic equilibrium is unstable, and there exists a unique stable endemic equilibrium with a steady state featuring no infections $\left(I_{i}(\infty)=I_{j}(\infty)=0\right)$ and shares of susceptible agents $S_{i}(\infty) \in(0,1)$ and $S_{j}(\infty) \in(0,1)$ that satisfy equations (17) and (18).

If the global reproduction rate satisfies $\mathcal{R}_{0}>1$, there exists a unique asymptotically stable 'pandemic' equilibrium. Following a small initial infection, the share of worldwide infected households necessarily increases for a period of time, and then declines to a point at which infections vanish and the share of susceptible households in the population in each country $\left(S_{i}(\infty), S_{j}(\infty)\right)$ takes a value strictly between 0 and 1 . These steady-state levels of infections in the two countries satisfy the following system of non-linear equations

$$
\begin{aligned}
& \ln S_{i}(\infty)=-\frac{2 \alpha_{i} n_{i i}}{\gamma_{i}}\left(1-S_{i}(\infty)\right)-\frac{\alpha_{j} n_{i j}+\alpha_{i} n_{j i}}{\gamma_{j}}\left(1-S_{j}(\infty)\right) \\
& \ln S_{j}(\infty)=-\frac{2 \alpha_{j} n_{j j}}{\gamma_{j}}\left(1-S_{j}(\infty)\right)-\frac{\alpha_{j} n_{i j}+\alpha_{i} n_{j i}}{\gamma_{i}}\left(1-S_{i}(\infty)\right) .
\end{aligned}
$$

Totally differentiating this system of equations, the steady-state values of $S_{i}$ and $S_{j}$ are decreasing in $n_{i i}, n_{j j}, n_{i j}$, and $n_{j i}$, as shown in Online Appendix D.1. Combining these results with our comparative statics for bilateral interactions with respect to international trade and travel frictions $\left(d_{i j}, t_{i j}, t_{j i}, \mu_{i j}, \mu_{j i}\right)$ from the previous section, we thus obtain a characterization of the response of steady-state susceptibles to these trade and travel frictions, a characterization we will exploit in some of our results below.

\subsection{Epidemiological Externality}

We now highlight a distinctive implication of our international trade setting. We show that there is a powerful epidemiological externality between countries, which depends on the endogenously determined pattern of bilateral interactions. In particular, since $\mathcal{R}_{0}$ is nondecreasing in $n_{i j}$ and $n_{j i}$ in equation (16), we have:

$$
\mathcal{R}_{0} \geq\left.\mathcal{R}_{0}\right|_{n_{i j}=n_{j i}=0}=\max \left\{\frac{2 \alpha_{i} n_{i i}}{\gamma_{i}}, \frac{2 \alpha_{j} n_{j j}}{\gamma_{j}}\right\}
$$


Therefore, even if one country has the disease under control (a reproduction number $\mathcal{R}_{0 i}$ based only on its domestic interactions of less than one), it will necessarily participate in a global pandemic if there is positive trade between the countries, and the other country does not have the disease under control. ${ }^{18}$ Although we illustrate this result here for two countries, we show in Online Appendix D.4 that it holds more generally. With $N \geq 2$ countries and arbitrary country asymmetries, a sufficient condition for the spectral radius of the next generation matrix to be greater than one is that any country has a reproduction number $\mathcal{R}_{0 i}$ based only on its domestic interactions of greater than one. Note, however, that the intensity of these domestic interactions is lower in the open economy than in the closed economy, and is decreasing in the number of trade partners, because of substitution between domestic and foreign interactions. ${ }^{19}$

In Figure 2, we illustrate these analytical results by holding the infection rate in Country 1 $\left(\alpha_{1}\right)$ constant and varying the infection rate in Country $2\left(\alpha_{2}\right)$. The starting point is two identical countries with a common infection rate of $\alpha_{1}=\alpha_{2}=0.04$. The rest of the parameter values are described in Online Appendix K. For this initial common infection rate, the global reproduction number is $\mathcal{R}_{0}=0.75$, and the open economy domestic reproduction rates are $\mathcal{R}_{01}=\mathcal{R}_{02}=0.46$. As a result, the initial infection quickly dies out and there is no global pandemic. The fraction of recovered agents in the long run, $R_{i}(\infty)$, which is equal to the cumulative number of infected agents in the absence of deaths, is essentially zero in both countries.

Figure 2: The Impact of Changes in the Exogenous Infection Rate in Country 2, $\alpha_{2}$
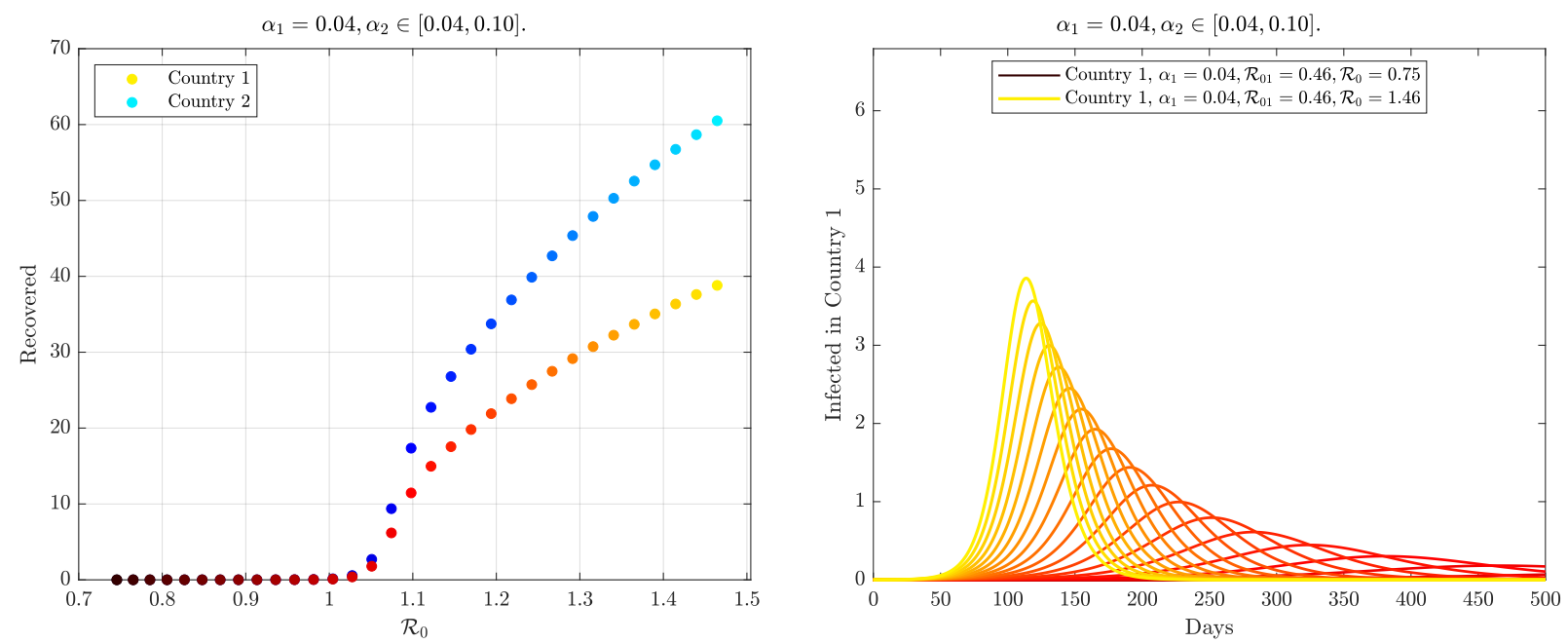

Note: See Online Appendix K for further details on the parameters and algorithms used in the numerical simulations. The color of each curve in the right panel corresponds to the colors of the points in the left panel.

The left panel of Figure 2 plots $R_{i}(\infty)$ as a function of $\mathcal{R}_{0}$ as we progressively increase $\alpha_{2}$ from 0.04 to 0.10 . The value of $\mathcal{R}_{0}$ is monotone in $\alpha_{2}$ and increases from 0.75 to 1.46 . Hence, as

\footnotetext{
${ }^{18}$ In Online Appendix D.3, we compute the largest positive eigenvalue of the Jacobian of the system around $S_{i}=S_{j} \simeq 1$ and $I_{i}=I_{j} \simeq 0$ and show that, consistent with equation (19), a pandemic-free equilibrium can only be locally stable whenever $2 \alpha_{i} n_{i i} / \gamma_{i} \leq 1$ and $2 \alpha_{j} n_{j j} / \gamma_{j j} \leq 1$.

${ }^{19}$ Note that, even if one country has a reproduction number $\mathcal{R}_{0 i}$ greater than one based on its domestic interactions, the resulting global pandemic can be small if that country is small relative to other countries.
} 
the exogenous infection rate of Country 2 increases, the global reproduction rate increases beyond the critical value of 1 , and the world experiences a global pandemic. Note how the fraction of the cumulative number of recovered agents rises rapidly once $\mathcal{R}_{0}$ increases beyond 1 and both countries go through increasingly severe pandemics. Note also the importance of cross-country contagion in the open economy. Even though nothing is changing in the domestic characteristics of Country 1, it is dramatically affected by the worsening conditions in Country 2 through the epidemiological externality. The right panel shows the evolution of the pandemic in Country 1 for different levels of severity of the disease environment in Country 2. The most severe and rapid pandemics are associated with the highest values of $\alpha_{2}$ (the lightest curve in the graph). As $\alpha_{2}$ declines and $\mathcal{R}_{0}$ falls and crosses the value of 1 , the evolution of infections flattens and becomes longer, until the pandemic eventually disappears.

In existing multi-group SIR models, multiple waves of infection can occur in the presence of heterogeneity in hosts, pathogens, space, and/or time, as reviewed in Bo et al. (2020). As we show in Online Appendix D.5, in our international trade setting, multiple waves of infection occur when the two countries have very different timings for their own initial waves of infections, shaped by their endogenous trade interactions, and when these two countries are sufficiently asymmetric in size. In contrast, if the timing of disease spread is sufficiently similar across countries, or if these countries are of similar size, both countries end up with a synchronized pandemic with only one peak. This pattern of multiple waves of infections between interconnected locations was observed during the Covid-19 pandemic. Although these multiple waves in part reflected time-varying policies such as lockdowns, there was also much discussion of countries (or states in large countries such as the United States) becoming reinfected from one another. ${ }^{20}$

\subsection{Trade Integration and Pandemics}

We now highlight two further distinctive predictions of our framework relative to conventional multigroup SIR models, which again follow from the fact that bilateral interactions are endogenous to international trade and travel frictions. In terms of the stability of a pandemic-free equilibrium, inspection of equation (19) might lead one to infer that avoiding a pandemic is always more difficult in a globalized world. First, for given positive values of $n_{i i}$ and $n_{j j}$, if the ratio $\alpha_{i} / \gamma_{j}$ is sufficiently high in any country in the world, a global pandemic affecting all countries cannot be avoided, even though the country with the lower ratio $\alpha_{i} / \gamma_{j}$ might well have avoided it under autarky. Second, even when $\alpha_{i}=\alpha_{j}$ and $\gamma_{i}=\gamma_{j}$, the max operator in (19) may seem to imply that the pandemic-free equilibrium is less likely to be stable in the open economy.

However, this reasoning does not take into account that the intensities of bilateral interactions $\left(n_{i i}, n_{j j}, n_{i j}, n_{j i}\right)$ are endogenous to trade integration. On the one hand, if countries are symmetric, lower trade costs increase the overall number of interactions (domestic plus foreign), as shown in Proposition B.3 in Online Appendix B.5. This increase in overall interactions acts to

\footnotetext{
${ }^{20}$ See, for example, the discussion of U.S. regional patterns of infection in the Covid -19 pandemic in the New York Times: "What Previous Covid-19 Waves Tell Us About the Virus Now".
} 
promote disease diffusion. On the other hand, domestic and foreign interactions are substitutes in the constant elasticity gravity equation, which implies that lower trade costs induce substitution from domestic to foreign interactions, as shown in Proposition B.2 in Online Appendix B.4. This substitution could either increase or decrease disease diffusion, depending on the disease environment in each country $\left(\alpha_{i}, \alpha_{j}, \gamma_{i}, \gamma_{j}\right)$, as determined by local culture, administrative capacity, and medical technology.

We now show formally that trade integration can either increase or decrease the range of parameters where a pandemic occurs and the severity of the pandemic if it does occurs. We begin by considering a fully symmetric world in which all primitives of the model (population size, technology, trade barriers, recovery rates, etc.) are common in both countries, so that we have $n_{d o m} \equiv n_{i i}=n_{j j}$, $n_{\text {for }} \equiv n_{i j}=n_{j i}, \alpha_{i}=\alpha_{j}=\alpha$, and $\gamma_{i}=\gamma_{j}=\gamma$. In such a case, $\mathcal{R}_{0}$ simplifies to

$$
\mathcal{R}_{0}=\frac{2 \alpha\left(n_{\text {dom }}+n_{\text {for }}\right)}{\gamma},
$$

and it thus follows immediately from Proposition B.3 in Online Appendix B.5 that a decline in any (symmetric) international trade friction increases $\mathcal{R}_{0}$, and thus decreases the range of parameters for which a pandemic-free equilibrium is stable. Furthermore, in this same symmetric case, the steady-state share of susceptible households in the population is identical in both countries and implicitly given by

$$
\ln S_{i}(\infty)=-\frac{2 \alpha\left(n_{\text {dom }}+n_{\text {for }}\right)}{\gamma}\left(1-S_{i}(\infty)\right) .
$$

Thus, not only the frequency but also the severity of the pandemic is higher the lower are (symmetric) trade frictions. We summarize these results as follows:

Proposition 2 Suppose that countries are symmetric, in the sense that $L_{i}=L, Z_{i}=Z, \Upsilon_{i j}=\Upsilon$, $\alpha_{i}=\alpha_{j}$, and $\gamma_{i}=\gamma$ for all $i$. Then, a decline in any (symmetric) international trade friction: (i) increases $\mathcal{R}_{0}$, thus decreasing the range of parameters for which a pandemic-free equilibrium is stable, and (ii) increases the share of each country's population that becomes infected during the pandemic when $\mathcal{R}_{0}>1$.

Although we have so far focused on a fully symmetric case, the main results in this Proposition continue to hold true even if countries are not perfectly symmetric. More generally, a necessary condition for the pandemic-free equilibrium to be stable is $\mathcal{R}_{0}<1$, and thus what matters for the effects of reductions in trade and travel frictions is whether $\mathcal{R}_{0}<1$ increases or decreases with those reductions in barriers.

Figure 3 illustrates Proposition 2 for a case in which we introduce an asymmetry in the exogenous infection rate across countries but $\mathcal{R}_{0}$ is still decreasing in international trade frictions. We let $\alpha_{1}=0.04$ and $\alpha_{2}=0.07$, and study the cumulative number of recovered agents when we increase symmetric international trade frictions $\left(t_{i j}\right.$, left panel) and travel frictions ( $\mu_{i j}$, right panel). The first point on both graphs corresponds to $t_{12}=t_{21}=\mu_{12}=\mu_{21}=1$. The rest of the parameter values are described in Online Appendix K. The large infection rate in Country 2 generates a 
pandemic in both countries. Globalization is essential to generate this pandemic. As both graphs illustrate, as we increase either tariffs or travel restrictions, global interactions decline, and the total number of recovered agents decreases. Eventually, when the world is sufficiently isolated, the pandemic disappears and the pandemic-free equilibrium becomes stable. In both graphs, the value of $\mathcal{R}_{0}$ (plotted in orange and measured in the right axis) declines smoothly with frictions. The vertical line in the figure indicates the value of tariffs or travel frictions, respectively, corresponding to $\mathcal{R}_{0}=1 .{ }^{21}$

Figure 3: The Impact of Changes in Trade (left) and Travel (right) Frictions
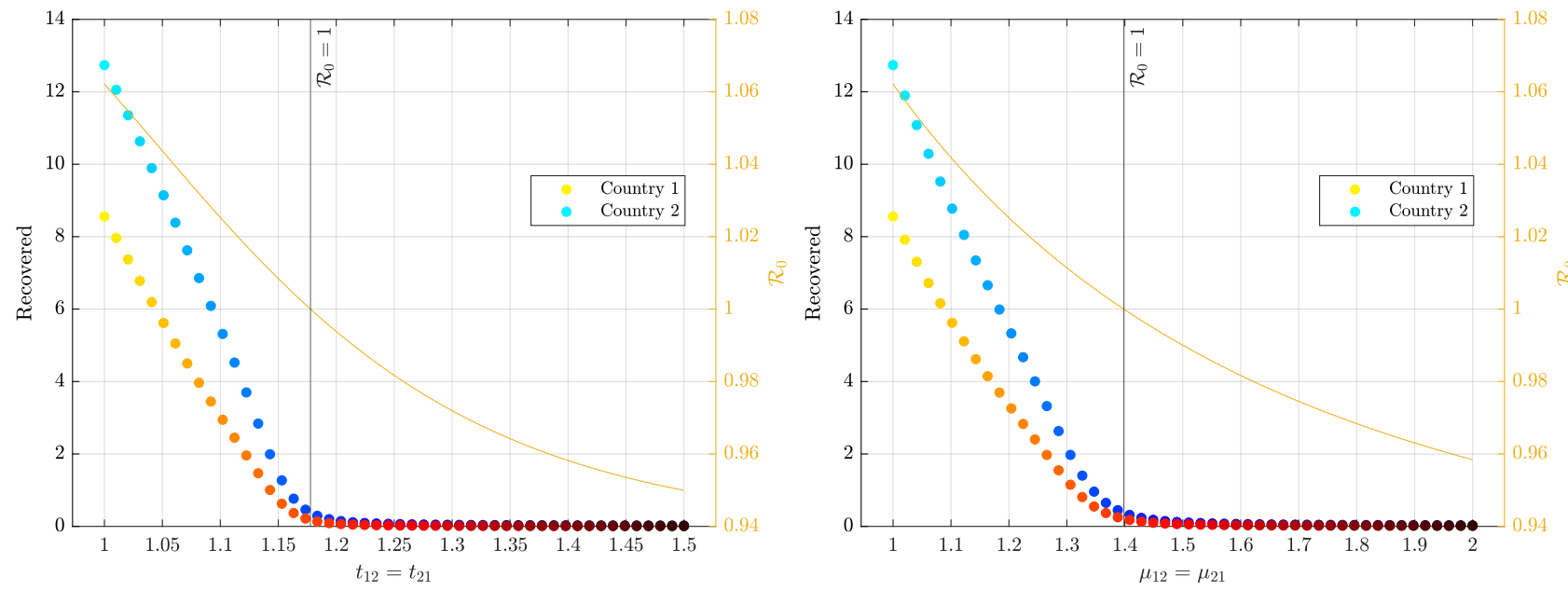

Note: See Online Appendix K for further details on the parameters and algorithms for these numerical simulations.

Although, in most cases, $\mathcal{R}_{0}$ increases as one lowers trade and travel frictions, we now show that lower trade costs can also reduce the risk of a pandemic if there are differences in the disease environment across countries (e.g., due to culture, administrative capacity or medical technology). Suppose, in particular, that country $j$ is a much lower risk environment, in the sense that $\alpha_{j}$ is very low - so infections are very rare - and $\gamma_{j}$ is very high - so infected households quickly recover in that country. In the limiting case $\alpha_{j} \rightarrow 0$, the condition that $\mathcal{R}_{0}<1$ reduces to ${ }^{22}$

$$
\frac{2 \alpha_{i} n_{i i}}{\gamma_{i}}+\frac{1}{\gamma_{j}} \frac{\left(\alpha_{i} n_{j i}\right)^{2}}{\gamma_{i}}<1 .
$$

For a high value of $\gamma_{j}$, it is then straightforward to see that the fall in country $i$ 's domestic interactions $n_{i i}$ associated with a reduction in international barriers makes this constraint laxer, even if $n_{j i}$ goes up with that liberalization. In those situations it is perfectly possible for a

\footnotetext{
${ }^{21}$ Note that the value of $R_{i}(\infty)$, does not become zero for either country right at the point where tariffs or travel frictions lead $\mathcal{R}_{0}$ to become greater than one. The reason is that even though one of the countries necessarily avoids a pandemic, it lingers close to its initial value of infections for a long time, which accumulates to a positive cumulative number of recovered agents.

${ }^{22}$ It is straightforward to show that a necessary condition for $\mathcal{R}_{0}<1$ is
}

$$
\frac{2 \alpha_{i} n_{i i}}{\gamma_{i}}+\frac{2 \alpha_{j} n_{j j}}{\gamma_{j}}-\frac{2 \alpha_{i} n_{i i}}{\gamma_{i}} \frac{2 \alpha_{j} n_{j j}}{\gamma_{j}}+\frac{\left(\alpha_{j} n_{i j}+\alpha_{i} n_{j i}\right)^{2}}{\gamma_{i} \gamma_{j}}<1
$$


pandemic-free equilibrium worldwide to only be stable when barriers are low. The intuition for this result is straightforward. In such a scenario, globalization makes it economically appealing for agents from a high-risk country to increase their interactions with agents in a low-risk country, and despite the fact that overall interactions by these agents may increase, the reduction in domestic interactions in their own high-risk environment is sufficient to maintain the disease in check.

More generally, beyond this limiting case, if there are sufficiently large differences in the disease environment across countries, lower trade costs can reduce both the range of parameters where a pandemic occurs and its severity when it does occur. We summarize this result as follows:

Proposition 3 When the contagion rate $\alpha_{i}$ and the recovery rate $\gamma_{i}$ vary sufficiently across countries, a decline in any international trade friction (i) decreases $\mathcal{R}_{0}$, thus increasing the range of parameters for which a pandemic-free equilibrium is stable, and (ii) when $\mathcal{R}_{0}>1$, it reduces the share of the population in the high-risk (high $\alpha_{i}$, low $\gamma_{i}$ ) country that becomes infected during the pandemic, and it may also reduce the share of the population in the low-risk (low $\alpha_{i}$, high $\gamma_{i}$ ) country that become infected during the pandemic.

Figure 4: The Impact of Changes in Trade (left) and Travel (right) Frictions with Large Differences in Infection Rates Across Countries $\left(\alpha_{1}=0.008\right.$ and $\left.\alpha_{2}=0.052\right)$
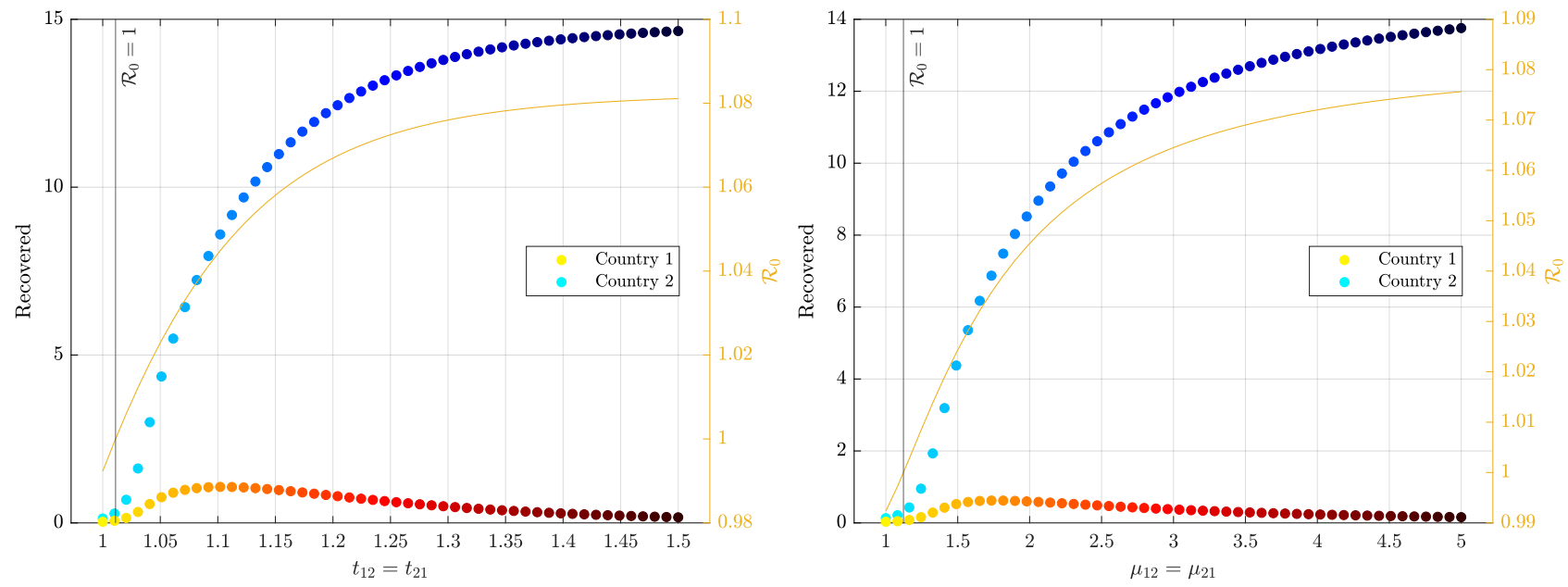

Note: See Online Appendix K for further details on the parameters and algorithms for these numerical simulations.

Figure 4 illustrates Proposition 3 by presenting examples in which increases in trade and travel barriers eliminate the possibility of a pandemic-free equilibrium. The figure considers the case of a small infection rate in the healthy country, Country 1 , of $\alpha_{1}=0.008$, and sets the infection rate in County 2 at a standard value of $\alpha_{2}=0.052 .{ }^{23}$ In both panels, increases in frictions now lead to increases in $\mathcal{R}_{0}$ (again depicted in orange and measured in the right axis). Without frictions the pandemic-free equilibrium is stable. Agents in Country 2 interact sufficiently with

\footnotetext{
${ }^{23}$ Relative to the baseline parameters in Online Appendix K, this example also lowers $c$ to 0.1 and $\phi$ to 1.5 . These additional changes increase the overall number of domestic and foreign interactions.
} 
the healthier Country 1, which helps them avoid the pandemic. As both economies impose more frictions, domestic interactions increase rapidly, while foreign interactions drop. This is bad news for Country 2, since its larger infection rate now leads to a pandemic. Perhaps surprisingly, it is also bad news for Country 1 since, although it interacts less with Country 2, it does so sufficiently to experience a pandemic. Larger frictions, which decrease aggregate income in both countries smoothly, also worsen the pandemic in both countries, at least when frictions are not too large; a clear case for free trade and mobility. Of course, as frictions increase further, eventually they isolate Country 1 sufficiently and so the severity of its local pandemic declines. In autarky, Country 1 avoids the pandemic completely, but at a large cost in the income of both countries. In contrast, higher frictions always worsen the pandemic in Country 2. Contacts with the healthy country are always beneficial, since they dilute interactions with locals, which are more risky.

This possibility for lower trade and travel costs to reduce the incidence and severity of pandemics arises from the substitutability between domestic and foreign interactions. In Online Appendix I, we show that this substitutability is implied by a constant elasticity gravity equation, in the sense that reductions in trade costs increase foreign interactions relative to domestic interactions. We also show empirically that a constant elasticity gravity equation provides a good approximation to observed travel behavior. In practice, it may be hard to find examples in which the differences in disease environment across countries are sufficiently large that reductions in trade and travel costs do indeed reduce the magnitude of pandemics. Furthermore, evaluating the impact of changes in trade and travel policies on disease diffusion is complicated by the fact that these policies are themselves endogenous to this disease diffusion. Still, some evidence from the Covid-19 pandemic is consistent with our predictions that (i) travel bans can trap individuals in 'hotspot' locations and exacerbate disease diffusion and (ii) localized lockdowns can be unsuccessful in containing the disease in the presence of spillovers from neighboring locations. Burlig et al. (2021) use data from domestic travel bans in several developing economies (India, China, Indonesia, the Philippines, South Africa, and Kenya) and show that travel bans can in some circumstances increase cumulative infections relative to a counterfactual without restrictions. ${ }^{24}$ In an empirical analysis of Chilean municipalities during the Covid-19 pandemic, Li et al. (2022) find that the success of localized lockdowns in controlling the transmission of this disease was heavily influenced by indirect effects from neighboring geographic areas. ${ }^{25}$

\section{General-Equilibrium Social Distancing}

In this section, we generalize our analysis to allow the disease to affect mortality and the ability to work and trade. For the time being, we assume however that agents are unaware of the source of

\footnotetext{
${ }^{24}$ In a similar vein, in reviewing the impact of travel bans during the early-phases of this pandemic, Grépin et al. (2021) find heterogeneity in their effects depending on how early they were implemented, and stress the importance of taking into account restrictions on domestic travel in addition to those on international travel.

${ }^{25}$ International evidence on the difficulty of completing sealing borders to eliminate disease transmission comes from the experience of Australia, New Zealand and China during the Covid-19 pandemic, as discussed in "Why Travel Bans are Usually the Wrong Way to Curb Omicron", The Economist, 2022/01/01.
} 
the infection, and hence have no incentive to change their individual behavior. This generalization introduces a further interaction between trade and disease dynamics, because changes in relative labor supplies give rise to a form of general equilibrium social distancing. In Subsection 5.1, we first introduce deaths. In Subsection 5.2, we next incorporate both deaths and reduced labor supply by infected workers.

\subsection{Deaths}

We begin by allowing the infection to affect mortality. We assume that infected agents die with probability $\eta_{i}$ and recover with probability $\gamma_{i}$, where both probabilities depend on the country in which infected agents reside, and not on where they were infected. ${ }^{26}$ Using this new assumption, the shares of households of each type evolve according to the following laws of motion (where we again ignore time subscripts to keep the notation tidy):

$$
\begin{aligned}
\dot{S}_{i} & =-2 n_{i i}(\mathbf{w}) \alpha_{i} S_{i} I_{i}-\left[n_{i j}(\mathbf{w}) \alpha_{j}+n_{j i}(\mathbf{w}) \alpha_{i}\right] S_{i} I_{j} \\
\dot{I}_{i} & =2 n_{i i}(\mathbf{w}) \alpha_{i} S_{i} I_{i}+\left[n_{i j}(\mathbf{w}) \alpha_{j}+n_{j i}(\mathbf{w}) \alpha_{i}\right] S_{i} I_{j}-\left(\gamma_{i}+\eta_{i}\right) I_{i} \\
\dot{R}_{i} & =\gamma_{i} I_{i} \\
\dot{D}_{i} & =\eta_{i} I_{i}
\end{aligned}
$$

There are two main differences between this dynamic system and that in equations (13)-(15). First, we now have four types of agents, as some infected agents transition to death rather than recovery. Second, we now need to make explicit the dependence of the contact rates $n_{i i}(\mathbf{w}), n_{i j}(\mathbf{w})$ and $n_{j i}(\mathbf{w})$ on the vector of equilibrium wages $\mathbf{w}$. As the changes in each country's population caused by deaths affect wages, this changes the intensity of bilateral interactions, which now evolve endogenously over the course of the pandemic. In particular, the equilibrium wage vector is determined by the following goods market clearing condition:

$$
\sum_{j \in \mathcal{J}} \pi_{j i}(\mathbf{w}) w_{j}\left(1-D_{j}\right) L_{j}=w_{i}\left(1-D_{i}\right) L_{i}
$$

where recall that $\pi_{i j}(\mathbf{w})$ and $n_{i j}(\mathbf{w})$ are now given by equations (9) and (12), respectively.

In the special case in which $\eta_{i}=0$, the system with deaths in equations (20)-(23) and equation (24) naturally reduces to the system without deaths in equations (13)-(15). Furthermore, the general equilibrium system with deaths is continuous in $\eta_{i}$, because (i) $D_{i}$ is continuous in $\eta_{i}$ given wages; (ii) $\pi_{i j}(\mathbf{w})$ is continuous in $\mathbf{w}$ for all $i j$ from equation (9); (iii) $\mathbf{w}$ is continuous in $D_{i}$ from equation (24); and (iv) $n_{i j}(\mathbf{w})$ is continuous in $\mathbf{w}$ for all $i j$ from equation (12). This property of the continuity of the entire general equilibrium system in $\eta_{i}$ ensures that all of the results established for our baseline model in the previous section necessarily hold in this generalization for sufficiently small $\eta_{i}$.

We now show that the impact of deaths on labor supply and equilibrium wages introduces a

\footnotetext{
${ }^{26}$ We implicitly assume that if one of the household members dies, the other one does too.
} 
form of general equilibrium social distancing into the model. First, the decline in a country's labor supply from mortality leads to a change in its relative equilibrium wage (a terms of trade effect). Second, this decline in labor supply from mortality reduces the range of goods available in that country (a decline in the quantity of varieties produced). In an interior equilibrium in which agents source a subset of the varieties produced in each country, the measure of varieties sourced from each country is determined by its relative wage, as shown in Online Appendix E.1. Therefore, the relative wage is a summary statistic for all general equilibrium effects in such an interior equilibrium where agents source a subset of varieties.

Purely through this general equilibrium force of changes in relative labor supplies, agents in the healthy country engage in a form of endogenous social distancing, in which they skew their interactions away from the country with a worse disease environment, as summarized in the following proposition (see Online Appendix E.2 for a proof):

Proposition 4 If country $j$ experiences more deaths than country $i$, the resulting change in relative wages $\left(w_{j} / w_{i}\right)$ leads country $i$ to reduce its interactions with country $j$ and increase its interactions with itself (general equilibrium social distancing).

We provide an analytical characterization of the elasticity of a country's relative wage with respect to its own population (and hence with respect to deaths) in Online Appendix E.3. We show that this elasticity becomes larger in absolute value as the trade elasticity becomes smaller, such that a larger change in relative wages and the terms of trade is required to restore goods market equilibrium. Additionally, this elasticity becomes larger as the country's share of income derived from itself becomes smaller, such that it becomes more dependent on foreign markets. Finally, this elasticity is bounded above in absolute value by one, and converges to this largest absolute value as the trade elasticity converges to zero.

In interpreting Proposition 4, it is worth stressing that even if one of the countries has more favorable health parameters $\left(\alpha_{i}, \gamma_{i}\right)$ than the other one, which country has more infections (and hence more deaths) can change during the pandemic if the two countries' waves of infection are staggered in time. In the initial stages of the pandemic, one country may experience a larger relative reduction in its labor supply (leading to endogenous social distancing in the other country), while in the later stages of the pandemic the other country experiences a larger relative reduction in its labor supply (leading to the opposite pattern of endogenous social distancing).

Another straightforward implication of explicitly modeling deaths is that they naturally affect aggregate income in both countries. More specifically, whenever changes in trade or travel barriers affect population, aggregate real income $\left(w_{i} L_{i} / P_{i}\right)$ and aggregate welfare $\left(W_{i} L_{i}\right)$ are directly impacted by trade-induced changes in population. Because around $\mathcal{R}_{0}=1$ deaths are particularly responsive to changes in trade frictions (as evident in Figures 3 and 4), changes in trade frictions can have substantial effects on welfare through deaths relative to their conventional effects through welfare gains from goods trade.

In Figure 5, we illustrate the results in Proposition 4 with a numerical example. For illustration purposes, we consider a case with extreme differences in death rates, in which Country 1 has 
Figure 5: General Equilibrium Induced Social Distancing
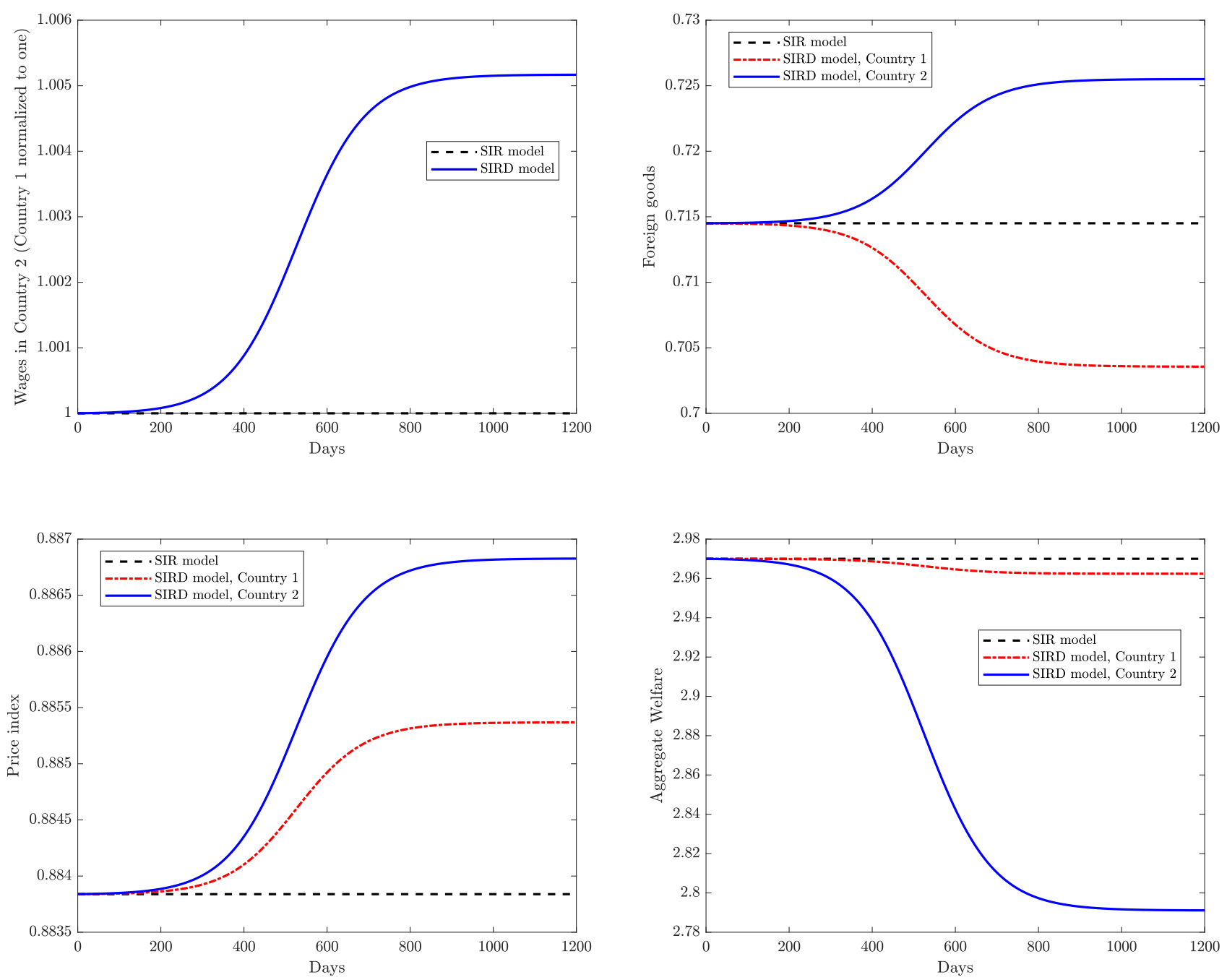

Note: See Online Appendix K for further details on the parameters and algorithms for these numerical simulations.

a death rate of 1 percent $\left(\eta_{1} /\left(\eta_{1}+\gamma_{1}\right)=0.01\right)$ and Country 2 has a death rate of 50 percent $\left(\eta_{2} /\left(\eta_{2}+\gamma_{2}\right)=0.50\right)$. The rest of the parameters are set to their baseline, symmetric values, across countries. We denote our generalization with deaths by 'SIRD model' and indicate our baseline specification with no deaths $\left(\eta_{1}=\eta_{2}=0\right)$ by 'SIR model.' The larger death rate in Country 2 leads to a fall in its relative labor supply, which increases relative wages, as shown in the top-left panel. Since the countries are otherwise symmetric, and we chose the wage of Country 1 as the numéraire, only the wage of Country 2 rises above one when deaths occur. The resulting increase in Country 2's relative wage is small (0.5\%), even though about $6 \%$ of agents end up dying in Country 2. Labor supply falls, but so does the aggregate demand for goods in that country and the set of varieties it produces, and the two countries here have identical size.

The rise in the relative wage of Country 2 implies that both countries tilt their consumption towards Country 1's varieties. Therefore, the consumption of foreign varieties increases in Country 
2 but falls in Country 1, as illustrated in the top-right panel. We see the opposite pattern for domestic varieties, although the adjustments are smaller. Ultimately, agents in both countries consume less varieties, which increases the price index in both countries, although by more in Country 2, as shown in the bottom-left panel. Real income falls in Country 1, both per capita and in aggregate, because of this increase in the price index. In contrast, in Country 2, real income per capita rises, because the wage increases by more than the price index. Nevertheless, aggregate real income falls as result of the reduction in labor supply from deaths, as shown in the bottom-right panel.

This general equilibrium social distancing prediction assumes that agents are unaware of the source of infection, and hence do not change their individual behavior, which may be more plausible for some diseases than others. Furthermore, the ability to detect this general equilibrium social distancing prediction empirically is likely to depend on the death rate, which again varies across diseases. Nevertheless, a major strand of research in the economic history literature argues that the decline in Europe's population of around one third as a result of the Black Death increased labor's relative bargaining power, which led to higher wages, and the institutional transition from feudalism to capitalism (see in particular North and Thomas, 1973, and Postan, 1973). Using timeseries data for England, Italy and Spain, Jedwab et al. (2022) show that the population declines from the Black Death were followed by sharp increases in real wages and real per capita GDP (see Figure 4 in that paper). Using U.S. data from the 1918 influenza pandemic, Garrett (2009) finds that states and cities with higher influenza and WW1 combat mortalities experienced statistically significantly higher manufacturing wage growth from 1914-19, even after controlling for wartime production and initial capital and value-added per capita in 1914. More broadly, looking beyond pandemics, there is substantial evidence of negative terms of trade effects from increased factor supplies, including for example Acemoglu and Ventura (2002) and Debaere and Lee (2004).

\subsection{Deaths and Reduced Worker Productivity}

We now further generalize our analysis to allow the pandemic to affect a country's labor supply through both deaths and a reduction in the fraction of time that infected individuals can work. We continue to assume that agents are unaware of the source of the infection, and hence have no incentive to change their individual behavior. Specifically, we assume that infected agents only provide $1-\varsigma_{i}>0$ units of labor. Furthermore, sick individuals isolate when they do not work, and so their interactions are proportional to their labor supply. Using this assumption, the shares of households of each type evolve according to the following modified laws of motion (where, as before, we ignore time subscripts to keep the notation tidy):

$$
\begin{aligned}
\dot{S}_{i} & =-2 n_{i i}(\mathbf{w}) \alpha_{i} S_{i}\left(1-\varsigma_{i}\right) I_{i}-\left[n_{i j}(\mathbf{w}) \alpha_{j}+n_{j i}(\mathbf{w}) \alpha_{i}\right] S_{i}\left(1-\varsigma_{j}\right) I_{j} \\
\dot{I}_{i} & =2 n_{i i}(\mathbf{w}) \alpha_{i} S_{i}\left(1-\varsigma_{i}\right) I_{i}+\left[n_{i j}(\mathbf{w}) \alpha_{j}+n_{j i}(\mathbf{w}) \alpha_{i}\right] S_{i}\left(1-\varsigma_{j}\right) I_{j}-\left(\gamma_{i}+\eta_{i}\right) I_{i} \\
\dot{R}_{i} & =\gamma_{i} I_{i} \\
\dot{D}_{i} & =\eta_{i} I_{i}
\end{aligned}
$$


Note that, since now an individual's reduced labor supply also results in reduced infections, a higher $\varsigma_{i}$ flattens the infection curve and reduces the total number of infections. In the limit, when $\varsigma_{1}=1$ in all countries, there is no pandemic since the initially infected isolate completely and infections do not spread. The modified goods market clearing condition becomes

$$
\sum_{j \in \mathcal{J}} \pi_{j i}(\mathbf{w}) w_{j}\left(1-D_{j}-\varsigma_{j} I_{j}\right) L_{j}=w_{i}\left(1-D_{i}-\varsigma_{i} I_{i}\right) L_{i}
$$

where $\pi_{i j}(\mathbf{w})$ and $n_{i j}(\mathbf{w})$ are given by equations (9) and (12), respectively.

The term $L_{i}^{w f} \equiv\left(1-D_{i}-\varsigma_{i} I_{i}\right) L_{i}$ represents the total labor supply in country $i$, which is given by the total initial population, $L_{i}$, minus the number of deaths, $D_{i} L_{i}$, minus the time that infected agents do not work, $\varsigma_{i} I_{i} L_{i}$. The last term is new and implies that relatively high infections in a country will reduce its relative labor supply, which results in higher relative wages in that country. As with deaths, the larger relative wages generate a general equilibrium social distancing effect that makes individuals in the relatively healthier country interact less with those in the country with a high number of infections. A key difference is that the reduction in worker productivity while infected is only temporary for those workers who recover from the infection. Hence, these effects can be particularly large and rich when countries' waves of infections are not synchronized.

In Figure 6, we simulate the same example as in Figure 5 above, but vary $\varsigma_{i}$ from 0 to 0.05 for all $i$. The lightest curves present the case when $\varsigma_{i}=0$ and so they reproduce the exercise in Figure 5 , for comparison purposes. The three panels in Figure 6 show the wave of infections in each country, the relative wage in Country 2, and the relative labor supply in Country 2, respectively. Clearly, the main effect of an increase in $\varsigma_{i}$ is to reduce the pandemic in both countries, which reduces the labor supply effect and therefore the impact on relative wages. As people work less and isolate more, the pandemic is smaller, and so is the general equilibrium social distancing effect.

Figure 6: Evolution of Infections, Relative Wages, and Labor Supply Share with Reduced Labor Supply by the Infected
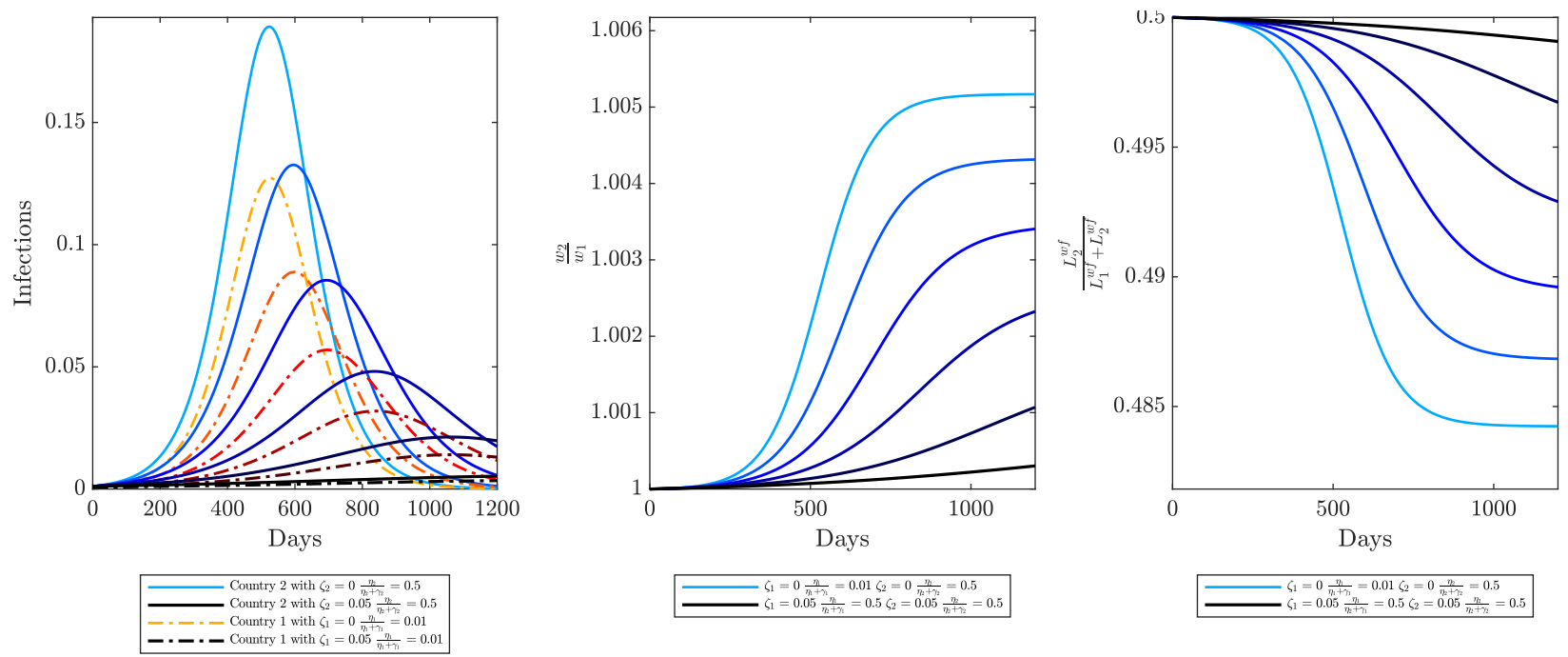

Note: See Online Appendix K for further details on the parameters and algorithms for these numerical simulations. 
In Online Appendix E.4 we study a case in which the pandemic again affects labor supply through reductions in the fraction of time sick agents can work, but where the infected do not isolate, and hence keep interacting with others. A natural interpretation of this case is a reduction in the productivity of the infected. This case allows us to underscore the subtle impact of the reduction in the labor supply of the infected on relative wages and interactions, without its direct effect on the magnitude of the pandemic through the self-isolation of the infected. As with deaths, a reduced labor supply by the infected generates a general equilibrium social distancing effect, but one that evolves according to the relative number of infected agents across countries. If the health environment in one country is worse than in the other, such that the pandemic is asynchronous across countries, this leads to changes in the countries' relative labor supplies and wages. Agents buy less from the country with temporarily higher relative wages, which lowers the peak in the number of infected agents in both countries.

\section{Behavioral Responses}

We next consider a further generalization of our baseline open-economy SIR model, in which we assume that the infection affects mortality and agents are aware of this. This generalization introduces a further interaction between trade and disease dynamics, because agents now adjust their individual behavior to the threat of infection.

Modeling these behavioral responses in an open-economy environment is challenging. Agents in each country choose profiles of time-varying interactions for each separate market. These profiles must be individually rational, in the sense that they are consistent with the conditions for dynamic optimization at the individual level, given the aggregate SIR disease dynamics and the path of the model's endogenous variables. But the aggregate SIR disease dynamics are themselves influenced by these individual profiles of time-varying interactions, as well as the path of the model's endogenous variables. Furthermore, the path of the model's endogenous variables is also shaped by both the aggregate SIR dynamics (through general equilibrium social distancing) and by the individual profiles of time-varying interactions (through behavioral responses). The interactions between these three sets of forces are particularly complex in the open economy, because of the much richer SIR disease dynamics with multiple countries, the more subtle general equilibrium interactions between countries, and the dimensionality of the state space at the individual level. We are not aware of any other research that analyses behavioral responses in models that feature both economic choices for spatial interactions and epidemiological disease dynamics in an open-economy setting.

To overcome these challenges, we build on the closed-economy specification of Farboodi et al. (2021), in which all infected individuals are assumed to be asymptomatic, in the sense that household behavior is independent of their specific health status, though their actual behavior is shaped by their expectation of the probability with which they are susceptible, infected, or recovered. How is that expectation formed? A natural assumption is that agents have rational expectations and that their belief of the probability with which they have a specific health status 
is equal to the share of the population in their country with that particular health status. ${ }^{27}$

\subsection{Theoretical Framework with Behavioral Responses}

We denote the individual beliefs of the probability of being infected, susceptible recovered, or dead with lowercase letters, except for their belief of the probability of being dead at time $t$, which we denote by $k_{i}(t)$ (instead of $\left.d_{i}(t)\right)$ to avoid a confusion with the notation we used for distance. The maximization problem of the individual, for known $i_{i}(0), s_{i}(0)$ and $k_{i}(0)=0$, is given by

$$
\begin{array}{cl}
W_{i}^{s}(0)=\max _{n_{i i}(\cdot), n_{i j}(\cdot)} & \int_{0}^{\infty} e^{-\xi t}\left[\left[Q_{i}\left(n_{i i}(t), n_{i j}(t)\right)-C_{i}\left(n_{i i}(t), n_{i j}(t)\right)\right]\left(1-k_{i}(t)\right)\right] d t \\
\text { s.t. } \quad & \quad \dot{s}_{i}(t)=-s_{i}(t)\left[\left(\alpha_{i} n_{i i}(t)+\alpha_{i} n_{i i}^{*}(t)\right) i_{i}(t)+\left(\alpha_{j} n_{i j}(t)+\alpha_{i} n_{j i}^{*}(t)\right) i_{j}(t)\right], \\
\dot{i}_{i}(t)= & s_{i}(t)\left[\left(\alpha_{i} n_{i i}(t)+\alpha_{i} n_{i i}^{*}(t)\right) i_{i}(t)+\left(\alpha_{j} n_{i j}(t)+\alpha_{i} n_{j i}^{*}(t)\right) i_{j}(t)\right] \\
& -\left(\gamma_{i}+\eta_{i}\right) i_{i}(t), \\
\dot{k}_{i}(t)= & \eta_{i} i_{i}(t),
\end{array}
$$

where $\xi$ is the rate of time preference, and where $\left[Q_{i}(\cdot, \cdot)-C_{i}(\cdot, \cdot)\right]$ takes the same form as in equation (6) above.

Note that we denote with an asterisk variables chosen by other households that affect the dynamics of infection of a given household. ${ }^{28}$ Implicitly, we are assuming that agents decide their optimal path of $n_{i i}(\cdot)$ and $n_{i j}(\cdot)$ at period zero and commit to following it. Otherwise, without commitment, at some future period and conditional on being alive, agents would want to reoptimize their choices by solving the problem above but setting $k_{i}(t)=0 .{ }^{29}$

From the present-value Hamiltonian, the optimality condition for the choice of $n_{i j}$ is

$$
\left[\frac{\partial Q_{i}\left(n_{i i}(t), n_{i j}(t)\right)}{\partial n_{i j}(t)}-\frac{\partial C_{i}\left(n_{i i}(t), n_{i j}(t)\right)}{\partial n_{i j}(t)}\right]\left(1-k_{i}(t)\right) e^{-\xi t}=\left[\theta_{i}^{s}(t)-\theta_{i}^{i}(t)\right] s_{i}(t) \alpha_{j} i_{j}(t),
$$

where $\theta_{i}^{s}$ and $\theta_{i}^{i}$ are the co-state variables for the changes in susceptibles $\left(\dot{s}_{i}\right)$ and infected $\left(\dot{i}_{i}\right)$, respectively, and we use $\theta_{i}^{k}$ to denote the corresponding co-state variable for the change in deaths

\footnotetext{
${ }^{27}$ Although households do not observe their own health status, they can form rational expectations about the share of the population with each health status if they have common knowledge of the model's parameters and rational expectations about the path of the pandemic. For the latter, it suffices to assume that agents observe pandemicrelated deaths at the outbreak of the disease. More specifically, at $t=0$, notice from equation (23) that (i) $I_{i 0}$ can be obtained from $I_{i 0}=D_{0} \eta_{i}$ since $D_{-1} \simeq 0$; (ii) $R_{i 0} \simeq 0$; and (iii) $S_{i t}$ is then trivially $S_{i 0}=1-I_{i 0}-R_{i 0}-D_{i 0}$. With this initial condition, agents can solve for the future path of the pandemic using rational expectations.

${ }^{28}$ For instance, though the aggregate domestic rate of contact in $i$ is $2 \alpha_{i} n_{i i}$, a household has no control over how many buyers visit the household's seller, so the household only controls the rate $\alpha_{i} n_{i i}$ of contacts generated by the household's buyer.

${ }^{29}$ The reason for this is that the probability of deaths acts like non-exponential discounting in the value function solved by agents, and it is well-understood that non-exponential discounting creates a wedge between the solution of dynamic problems with and without commitment. Farboodi et al. (2021) bypass this issue by assuming that, instead of foregoing future utility when dying, agents pay a one-time utility cost (or value of life) at the moment they die. Note, however, that for small probabilities of death $k$ : $(1-k d t) \approx e^{-k d t}$. Hence, for empirically reasonable values of $k$, we have found that our solution under full commitment is close to the solution under the exponential approximation, which is time consistent.
} 
$\left(\dot{k}_{i}\right)$. The optimality conditions associated with the co-state variables are given by:

$$
\begin{aligned}
-\dot{\theta}_{i}^{s}(t) & =-\left[\theta_{i}^{s}(t)-\theta_{i}^{i}(t)\right]\left[\left(\alpha_{i} n_{i i}(t)+\alpha_{i} n_{i i}^{*}(t)\right) i_{i}(t)+\left(\alpha_{j} n_{i j}(t)+\alpha_{i} n_{j i}^{*}(t)\right) i_{j}(t)\right] \\
-\dot{\theta}_{i}^{i}(t) & =\eta_{i} \theta_{i}^{k}(t)-\left(\gamma_{i}+\eta_{i}\right) \theta_{i}^{i}(t) \\
-\dot{\theta}_{i}^{k}(t) & =-\left[Q_{i}\left(n_{i i}(t), n_{i j}(t)\right)-C_{i}\left(n_{i i}(t), n_{i j}(t)\right)\right] e^{-\xi t}
\end{aligned}
$$

Finally, the transversality conditions are as follows: $\lim _{t \rightarrow \infty} \theta_{i}^{i}(t) i_{i}(t)=0, \lim _{t \rightarrow \infty} \theta_{i}^{s}(t) s_{i}(t)=0$, and $\lim _{t \rightarrow \infty} \theta_{i}^{k}(t) k_{i}(t)=0$.

In equilibrium, aggregate consistency implies that $i_{i}(t)=I_{i}(t), s_{i}(t)=S_{i}(t)$, and $k_{i}(t)=$ $D_{i}(t)$. Namely, an individual's rational expectations about their probability of being infected, susceptible, or dead in each period equal the corresponding population shares. Finally, we complete our description of the general equilibrium of the model with the goods market clearing condition that determines wages

$$
\sum_{j \in \mathcal{J}} \pi_{j i}(\mathbf{w}, t) w_{j}(t)\left(1-D_{j}(t)\right) L_{j}=w_{i}(t)\left(1-D_{i}(t)\right) L_{i}
$$

A key difference from our baseline model in Section 4, and from our generalization to incorporate changes in labor supply in Section 5 , is that agents now adjust bilateral interactions $\left(n_{j i}(\mathbf{w}, t)\right)$ in response to the threat of the infection. Nevertheless, we are able to solve for the dynamic path of the economy numerically using a backward shooting algorithm, as discussed in Online Appendix K. This backward shooting algorithm involves a guess of the share of deaths and infections in each country in the far away future, when the pandemic is over. Given this guess, we solve for the dynamic path of the economy backward and forward, and then check that our solution is consistent with this guess. When countries are symmetric, we can simply guess a value very close to zero for infections in the far away future and make an initial guess for the steady-state number of deaths. We then iterate until we find the equilibrium value of steady state deaths in both countries. With more than one country and country asymmetries, the dynamic path of the economy not only depends on the guess of steady state deaths in each country, but is highly sensitive to the relative value of final infections, even if their level is very close to zero in the long run. This increases the numerical complexity of the problem significantly. Hence, it is challenging to solve the model for many parameter values or expand the number of countries in the open economy equilibrium with asymmetric countries.

From inspection of the agent's optimization problem above, when there is zero probability of death $\left(\eta_{i}=0\right.$ and hence $k_{i}(t)=0$ for all $\left.t\right)$, the optimal choice of $n_{i i}(\cdot)$ and $n_{i j}(\cdot)$ is independent of the pandemic, given wages. Additionally, with no impact of the pandemic on labor supply, the goods market clearing condition implies that wages are time invariant. Therefore, in the special case of zero probability of death, the model here reduces to our baseline model without behavioral responses in Section 4. More generally, the Hamiltonian is continuous in $\eta_{i}$ and concave in the controls, given the properties of $Q_{i}(\cdot)$ and $C_{i}(\cdot)$ under the maintained assumption that $\sigma>2$ and $\phi>1$. Using these properties, the evolution of $n_{i i}(\cdot)$ and $n_{i j}(\cdot)$ is continuous in $\eta_{i}$, as is the 
number of deaths $D_{i}(t)$, and hence the path of equilibrium wages. This reasoning implies that the evolution of the economy with behavioral responses when $\eta_{i}>0$ approaches smoothly the one without behavioral responses as $\eta_{i} \rightarrow 0$. Therefore, as in the previous section, all the results in our original model without deaths or behavioral responses apply to this much more complicated model with behavioral responses when $\eta_{i}$ is sufficiently small in each country.

With a positive probability of death $\eta_{i}>0$, this is a significantly more complicated general equilibrium system, but we are able to show analytically that the solution to this problem necessarily involves individual-level social distancing. In the absence of a pandemic, households equate the marginal utility from sourcing varieties from each location to the marginal cost of sourcing those varieties. During a pandemic, households internalize that the interactions involved in sourcing varieties expose them to infection, which leads them to reduce interactions until the marginal utility from those interactions exceeds the marginal cost, as summarized in the following lemma (proven in Online Appendix F.1).

Lemma 1 Along the transition path, $\theta_{i}^{s}(t)-\theta_{i}^{i}(t) \geq 0$ for all $t$, which implies:

$$
\frac{\partial Q_{i}\left(n_{i i}(t), n_{i j}(t)\right)}{\partial n_{i j}(t)}>\frac{\partial C_{i}\left(n_{i i}(t), n_{i j}(t)\right)}{\partial n_{i j}(t)}, \quad \text { as long as } I_{j}(t)>0 \text {. }
$$

An implication of this result is that the pandemic generically has a larger impact on foreign interactions than on domestic interactions. This implication can been seen by re-arranging the optimality condition (25) and substituting for the marginal utility and marginal cost for interactions to obtain

$$
\frac{1}{n_{i j}} \frac{n_{i j} q_{i j}^{\frac{\sigma-1}{\sigma}}}{\sum_{\ell \in \mathcal{J}} n_{i \ell} q_{i \ell}^{\frac{\sigma-1}{\sigma}}} Q_{i}=\frac{1}{n_{i j}} c \mu_{i j} d_{i j}^{\rho} n_{i j}^{\phi}+\frac{\left[\theta_{i}^{s}(t)-\theta_{i}^{i}(t)\right] s_{i}(t) \alpha_{j} I_{j}(t)}{\left(1-D_{i}(t)\right) e^{-\xi t}},
$$

where the term on the left-hand side is the marginal utility from interactions; the first term on the right-hand side is the marginal cost of interactions; and the second term on the right-hand side is the wedge capturing the threat of infection. As foreign interactions are generically a smaller share of the consumption index than domestic interactions, the fraction on the left-hand side is generically smaller for foreign interactions $(i \neq j)$. Therefore, as a pandemic emerges and the threat of infection becomes positive, a larger reduction in $n_{i j}$ is generically needed for foreign interactions, in order to raise the marginal utility on the left-hand side until it is equal to the marginal cost plus the positive wedge capturing the threat of infections on the right-hand side.

\subsection{Behavioral Responses with Symmetric Countries}

We now illustrate some of these implications of behavioral responses for the case of symmetric countries. We use the baseline parameters with $\alpha_{i}=0.1, \gamma_{i}+\eta_{i}=0.2$, and $\eta_{i} /\left(\eta_{i}+\gamma_{i}\right)=0.0062$ (a $0.62 \%$ death rate among those infected) for all $i$. We also show a specification with half the death rate of $\eta_{i} /\left(\eta_{i}+\gamma_{i}\right)=0.003$ for all $i$, as well as the case without behavioral responses from the previous section. As we choose the wage in one country as the numéraire, with symmetric 
countries, the relative wage is also equal to one and constant over time. In the absence of any behavioral responses, this constant relative wage implies that both the mass of varieties and price index are constant over time. In contrast, in the presence of behavioral responses, households reduce the intensity of their interactions in response to the threat of infection, which leads to changes in the mass of varieties and the price index over time.

Figure 7: Behavioral Responses with Symmetric Countries for Various Death Rates
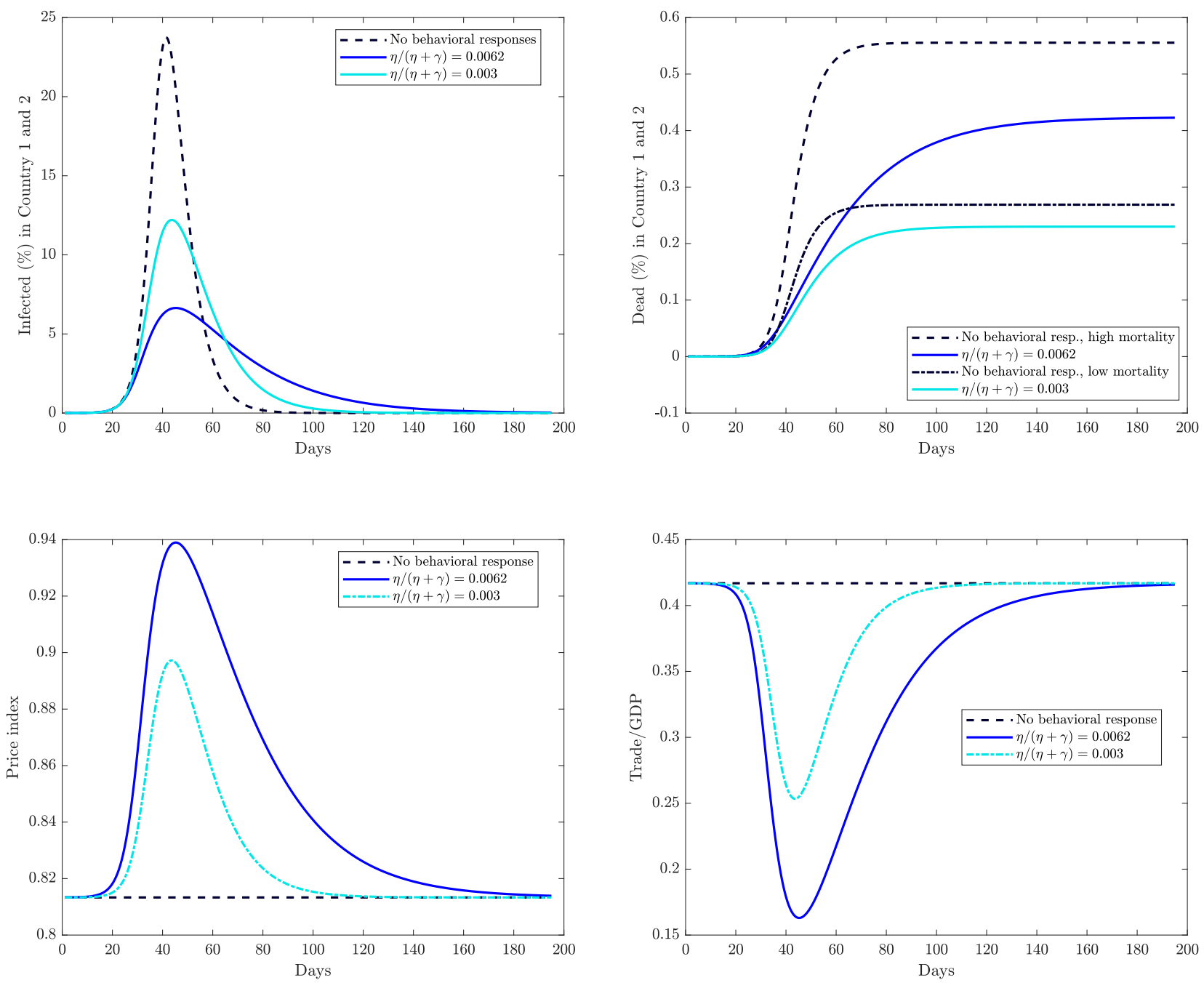

Note: See Online Appendix K for further details on the parameters and algorithms for these numerical simulations.

In the top-left panel of Figure 7, we show the percentage of individuals infected in Country 2 for all three specifications (with symmetry the figure for Country 1 is identical). Households' behavioral response of reducing interactions leads to a "flattening of the curve of the pandemic," such that the pandemic has lower peak and lower cumulative infections, but takes longer to subside. Clearly, the larger the death rate, the stronger the behavioral response and the flatter the resulting curve of infections. The top-right panel in Figure 7 presents the resulting evolution of cumulative deaths in Country 2. Behavioral responses delay and reduce total deaths, with the level (and proportional 
reduction) larger, the larger the death rate. Naturally, the behavioral response and the associated reductions in the number of deaths come at an economic cost for survivors. As the bottom-left panel shows, the reductions in the number of purchased domestic and foreign varieties increase the price index in each country, which results in a corresponding decline in real income. This increase in the price index, and reduction in real income, is larger the stronger the behavioral response, and hence is magnified by a higher death rate. Finally, the bottom-right panel displays the trade over GDP ratio (calculated as imports plus exports over GDP). In the example, trade/GDP falls from about 0.45 to less than 0.25 when the death rate is $0.3 \%$, and to 0.17 when the death rate is $0.62 \%$. Therefore, the flattening of the curve of infections and reduction in the number of deaths comes at the cost of lower trade and real income. Of course, behavioral responses are ex-ante privately optimal, so it is not surprising that they improve individual welfare. ${ }^{30}$

The value of mobility and trade frictions plays an important role in shaping the magnitude and pattern of behavioral responses. First, with symmetric countries, higher mobility and trade frictions imply a reduction in the overall volume of human interactions, which leaves less scope for behavioral responses. Second, higher mobility and trade frictions imply that more of the burden of adjustment falls on domestic rather than foreign transactions. In Figure 8, we show the evolution of the trade/GDP ratios for symmetric countries for two different levels of mobility (left panel) and trade (right panel) frictions and the baseline values of our other parameters. As discussed above, in the symmetric case without behavioral responses, all human contacts $n_{i i}(t)$ and $n_{i j}(t)$ are constant in time, which implies that mobility and trade frictions only reduce the level of the trade/GDP ratios. Once we incorporate behavioral responses, trade/GDP follows the trajectory of the pandemic. The larger value of trade frictions reduces trade openness, which dampens the absolute magnitude of the behavioral response, although trade openness can end up falling to quite low levels. In this example with $10 \%$ trade frictions, $\left(t_{12}=t_{21}=1.1\right)$, trade essentially falls to zero in the most severe phase of the pandemic. For each level of trade frictions, behavioral responses reduce the total number of deaths, and for the parameter values considered here, higher trade and mobility frictions also reduce the total number of deaths.

This theoretical prediction of a larger initial reduction in trade than in production also receives empirical support from the recent COVID-19 pandemic. In Figure J.1 in Online Appendix J, we show that world trade indeed fell faster than world production in the early stages of the pandemic, before later recovering. More generally, the prediction that behavioral responses lead agents to reduce interactions because of the threat of infection is supported by a range of empirical findings. Comparing consumer mobility across state and county boundaries with different levels of government-imposed COVID-19 restrictions, Goolsbee and Syverson (2020) find that most of the observed 60 percentage point decline in consumer traffic is explained by changes in individual be-

\footnotetext{
${ }^{30}$ Agents' responses are not in general socially optimal, because of the externalities between agents from the transmission of the disease. Fajgelbaum et al. (2021) characterizes the socially-optimal lockdown policies of a planner in a commuting model without private behavioral responses. Given the already rich interactions between globalization and disease transmission in our model with private behavioral responses, we leave the analysis of the optimal policies of the social planner in the presence of these private behavioral responses for future work.
} 
Figure 8: The Effect of Mobility and Trade Frictions on Trade/GDP with Behavioral Responses
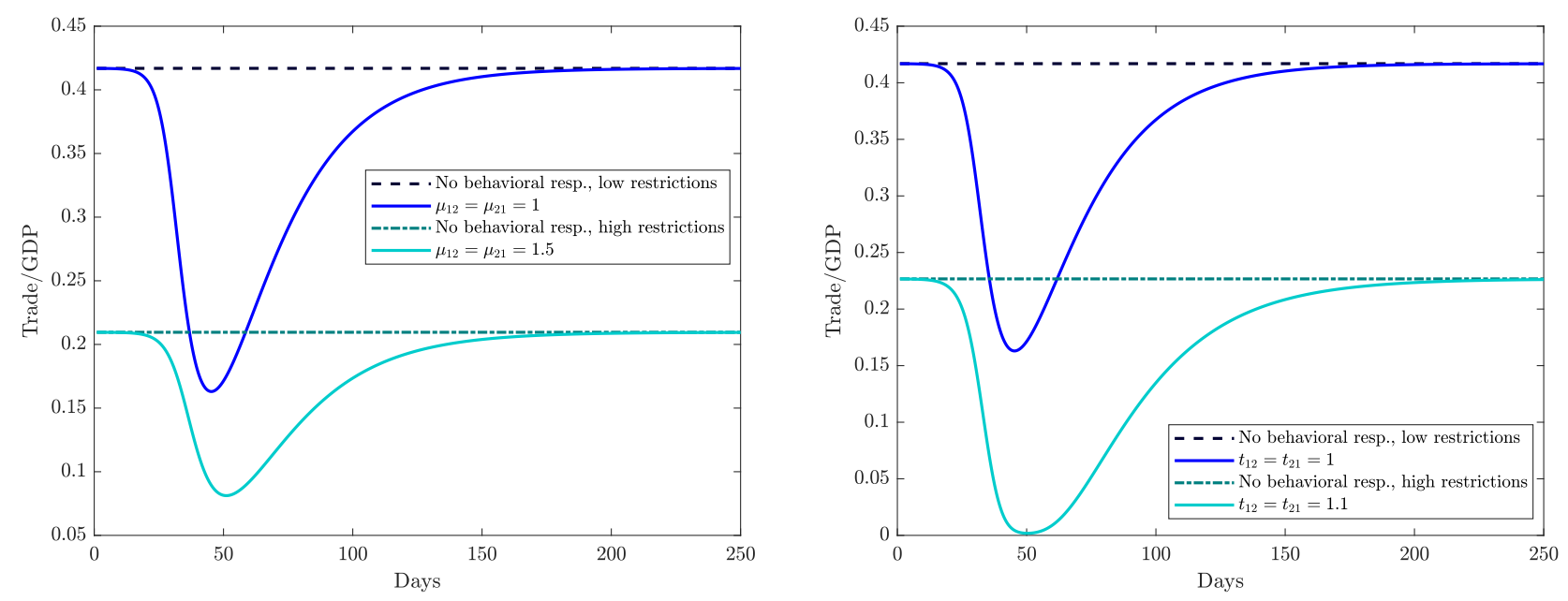

Note: See Online Appendix K for further details on the parameters and algorithms for these numerical simulations.

havior, whereas the contribution from government restrictions is only around 7 percent. Consumer traffic started dropping before the government restrictions were in place; was highly influenced by the number of COVID-19 deaths reported in the county; and showed a clear shift by consumers away from busier, more crowded stores toward smaller, less busy stores in the same industry. Using Swedish customs data, Akerman et al. (2022) compare firms exporting to foreign countries that were more versus less affected by COVID-19. Consistent with our theoretical predictions, firms exporting to more heavily-affected destinations experienced larger reductions in exports, and these reductions in firm exports exceeded the declines in total firm revenue. ${ }^{31}$

\subsection{Behavioral Responses with Asymmetric Countries}

Finally, we illustrate some of the implications of our model when countries are asymmetric. We focus on a case in which countries differ in their mortality rate, where remember that we assume that mortality is determined by the country in which a household lives rather than the country in which it was infected. We let Country 1 have a relatively low mortality rate of $0.3 \%$ and we leave the mortality rate of Country 2 at the higher baseline value of $0.62 \%$. Figure 9 presents the results. The top-left panel shows the percent of infections in each country. As benchmarks, we also display the average of infections in the two countries, as well as infections in the case of two symmetric countries with an average mortality rate of $0.46 \%$ (the mean of $0.3 \%$ and $0.62 \%$ ). There is a stronger behavioral response in the high-mortality Country 2 because households internalize the greater risk that infection leads to death, which results in a "flatter" curve of infections in this

\footnotetext{
${ }^{31} \mathrm{~A}$ number of recent papers have provided evidence of declines in bilateral trade following government-imposed lockdowns during the COVID-19 pandemic, including Aiyar et al. (2022), Berthou and Stumpner (2022), Brussevich et al. (2022), Cerdeiro and Komaromi (2020), Lagrogne-Joussier et al. (2021), and Liu et al. (2022), among others. The empirical results in Goolsbee and Syverson (2020) suggest that these 'lockdown effects' on trade may in part reflect behavioral responses that would have operated even in the absence government-mandated lockdowns.
} 
country. The low-mortality Country 1 ends up with about $10 \%$ higher total infections, because of its more subdued behavioral response. However, its lower mortality rate implies that it ends up with only about half the total number of deaths. This asymmetric behavioral response implies that Country 1 is a relatively dangerous destination for doing business in the early stages of the pandemic, but a relatively safe destination in the later stages of the pandemic, since it reaches herd immunity faster. Comparing the average response for the world with asymmetric countries to the response in the symmetric case with average mortality rates illustrates the implied aggregate effects from differences across countries in mortality rates. In the asymmetric case, the world's infection curve is marginally flatter than in a symmetric world with average mortality rates.

The top-right panel in Figure 9 displays Country 1's relative wage. As a result of the smaller behavioral response in this lower mortality country, there is a greater risk of infection in Country 1 in the early stages of the pandemic, which leads to a decline in demand for this country's varieties and a fall in its relative wage. Once Country 1's infection rate falls, demand for its varieties recovers, and hence so does its wage. Eventually, once Country 1's infection rate falls below that of Country 2 , it becomes the relatively safe environment in which to source varieties, and its relative wage rises temporarily above one, before falling back to one as the pandemic ends. Therefore, these behavioral responses in general equilibrium with asymmetric countries lead to demand effects that reduce the relative wage of the country with a relatively higher infection rate. In addition, as shown in the previous section, there is another general equilibrium effect from changes in relative labor supply. A country with a higher death rate experiences a reduction in its relative labor supply, which leads to an increase in its relative wage. The top-right panel of Figure 9 shows the balance of these forces, and demonstrates that relative demand effects generally dominate and overturn the result in Section 5 linking higher death rates to higher relative wages.

As before, the stronger behavioral response in Country 2 as a result of its higher mortality rate comes with greater economic costs. Country 2's reduction in domestic and foreign purchases raises its price index and reduces its real income. The effect on the price index in Country 1 is more nuanced. Country 1 also reduces domestic and foreign interactions, which tends to increase its price index. However, the decline in its relative wage during the first part of the pandemic reduces the price of domestic varieties. The bottom-left panel in Figure 9 shows how these forces result in a price index with multiple peaks. Overall, the effect of the pandemic on the real income of Country 1 is negative but substantially smaller in magnitude than in Country 2. As shown in the bottomright panel, the reduction in human interactions from social distancing reduces trade openness dramatically, particularly in Country 2, where behavioral responses are stronger. The asymmetry in mortality rates between the two countries initially leads to a larger reduction in trade openness than in a symmetric world with average mortality rates, in part because the behavioral response of Country 2 is particularly strong in the earlier phases of the pandemic. Later in the pandemic, the asymmetric case has higher trade openness than in a symmetric world, because the initially subdued behavioral response of Country 1 creates a more pronounced and faster wave of infections.

Despite the potential for significant disruptions in international trade during a pandemic, a 
Figure 9: Behavioral Responses with Asymmetric Mortality Rates
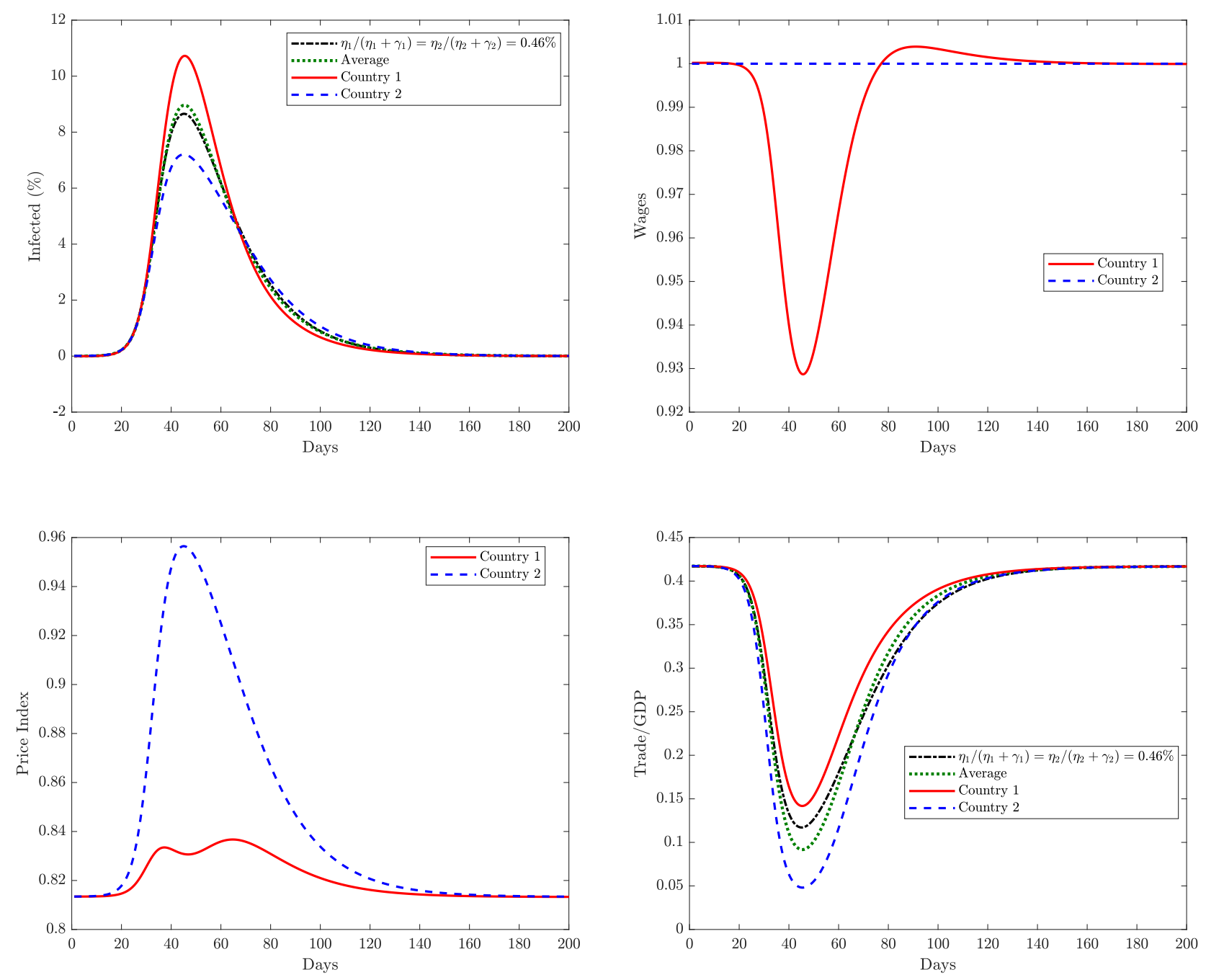

Note: See Online Appendix K for further details on the parameters and algorithms for these numerical simulations.

clear implication of the first-order condition (25) is that as long as $I_{i}(t)=I_{j}(t)=0$, human interactions are at the same level as in a world without the potential for pandemics. In other words, although there are rich dynamics of international trade during a pandemic, as soon as this pandemic is overcome (via herd immunity or the arrival of a vaccine), life immediately goes back to normal. In Online Appendix F.2, we examine the robustness of this prediction to the introduction of adjustment costs. Assuming that adjustment costs are symmetric, and the pandemic does not affect agents' beliefs of the probability of future pandemics, we continue to find a rapid V-shape recovery in economic activity and international trade flows after a global pandemic. 


\section{Conclusions}

Large-scale improvements in transportation technologies have dramatically increased the integration of the world economy and the ability of goods, people and infectious diseases to circulate around the globe. In this paper, we have developed a new theoretical framework to analyze the relationship between globalization and pandemics. Our framework incorporates two core mechanisms from economics and epidemiology. First, travel between countries transmits disease, as in the Susceptible-Infected-Recovered (SIR) model. Second, international trade stimulates travel between countries, according to a constant elasticity gravity equation mediated by mobility frictions.

Although these two mechanisms had been analyzed separately, we show that they interact in rich ways when considered jointly. First, we show that trade-motivated face-to-face interactions generate a powerful epidemiological externality across countries. A sufficient condition for a global pandemic to occur is that any country has a reproduction rate based on domestic interactions of greater than one, where the intensity of these domestic interactions differs between the open and the closed economies. Second, we have demonstrated that reductions in international frictions can either increase or decrease the range of parameter values for which a pandemic occurs. Third, if infections lead to deaths, or reduce individual labor supply, we have established the existence of a general equilibrium social distancing effect, whereby increases in relative prices in unhealthy countries reduce travel to those countries. Finally, we have studied the case in which agents internalize the threat of infection, and we have shown that agents' endogenous social distancing leads to a reduction in travel that is larger for higher-trade-cost locations, and hence leads to an initial fall in the ratio of trade to GDP in the early stages of the epidemic, followed by a swift

recovery. In the presence of adjustment costs, agents anticipate the costs incurred in adjusting contacts during a pandemic, but in practice we find that these anticipatory effects are small, at least for symmetric adjustment costs.

Although we have argued that our results are robust to various alternative specifications of our model of international trade, there remain a number of interesting areas for further research. For example, although we have explored dynamic variants of our model, we have not allowed international borrowing and lending to smooth out economic fluctuations caused by a pandemic. Similarly, our analysis has been positive in nature, but it would certainly be interesting to study the normative implications of our framework in future work. For example, the epidemiological externality in the model implies that whether a global pandemic occurs depends on the maximum reproduction rate based on domestic interactions across all countries. Therefore, if a vaccine is invented that is in limited supply in the short-run, in principle it could be in the interest of all countries to distribute that vaccine to the country with the most unhealthy domestic disease environment, in order to avoid a global pandemic. 


\section{References}

Acemoglu, Daron, Victor Chernozhukov, Iván Werning, Michael D. Whinston (2021), "Optimal Targeted Lockdowns in a Multi-Group SIR Model," American Economic Review: Insights, $3(4), 487-502$.

Acemoglu, Daron and Jaume Ventura (2002) "The World Income Distribution," Quarterly Journal of Economics, 117(2), 659-694.

Ahlfeldt, Gabriel, Stephen Redding, Daniel Sturm and Nikolaus Wolf (2015) "The Economics of Density: Evidence from the Berlin Wall," Econometrica, 83(6), 2127-2189.

Aiyar, Shekhar, Davide Malacrino, Adil Mohommad and Andrea Presbitero (2022), "International Trade Spillovers from Domestic COVID-19 Lockdowns," IMF Working Paper No. 2022/120.

Akerman, Anders, Karolina Ekholm, Torsten Persson, and Oskar N. Skans (2022) "Sweden's COVID-19 Recession: How Foreign and Domestic Infections Struck Against Firms and Workers," Stockholm University, mimeograph.

Alfaro, Laura, Ester Faia, Nora Lamersdorf, and Farzad Saidi (2020), "Social Interactions in Pandemics: Fear, Altruism, and Reciprocity," NBER Working Paper No. 27134.

Allen, Treb, and Costas Arkolakis (2014), "Trade and the Topography of the Spatial Economy," Quarterly Journal of Economics 129, no. 3: 1085-1140.

Allen, Treb, Costas Arkolakis, and Yuta Takahashi (2020), "Universal Gravity," Journal of Political Economy 128, no. 2: pp. 393-433.

Alvarez, Fernando, David Argente, and Francesco Lippi (2021), "A Simple Planning Problem for Covid-19 Lockdown, Testing, and Tracing " American Economic Review: Insights, 3(3), $367-82$.

Alvarez, Fernando, and Robert E. Lucas Jr. (2007), "General Equilibrium Analysis of the EatonKortum model of International Trade," Journal of Monetary Economics 54, no. 6, 1726-1768.

Anderson, James E. and Eric Van Wincoop (2003), "Gravity with Gravitas: A Solution to the Border Puzzle," American Economic Review, 93(1), pp.170-192.

Argente, David O., Chang-Tai Hsieh, and Munseob Lee (2022), "The Cost of Privacy: Welfare Effect of the Disclosure of Covid-19 Cases," Review of Economics and Statistics, 104(1), 176-186.

Arkolakis, Costas, Arnaud Costinot, and Andrés Rodríguez-Clare (2012), "New trade models, same old gains?" American Economic Review 102.1: 94-130.

Atkeson, Andrew (2020), "What Will Be the Economic Impact of Covid-19 in the US? Rough Estimates of Disease Scenarios," NBER Working Paper No. 26867.

Benedictow, Ole K. (2004) The Black Death 1346-1353: The Complete History, Woodbridge: The Boydell Press. 
Bernard, Andrew B., Andreas. Moxnes and Yukiko Saito (2019) "Production Networks, Geography and Firm Performance", Journal of Political Economy, 127(2), 639-688.

Berthou, Antoine and Sebastian Stumpner (2022), "Trade Under Lockdown," Working Paper 867, Banque de France.

Birge, John R., Ozan Candogan, and Yiding Feng (2020), "Controlling Epidemic Spread: Reducing Economic Losses with Targeted Closures," Becker-Friedman Institute Working Paper No. 2020-57.

Bisin, Alberto, and Andrea Moro (2021), "Learning Epidemiology by Doing: The Empirical Implications of a Spatial SIR Model with Behavioral Responses," Journal of Urban Economics, 103368 .

Blonigen, Bruce A. and Anca D. Cristea (2015), "Air Service and Urban Growth: Evidence from a Quasi-Natural Policy Experiment," Journal of Urban Economics 86, 128-146.

Boerner, Lars and Battista Severgnini (2014) "Epidemic Trade," Economic History Working Papers, 212, London School of Economics.

Brauer, Fred, and Carlos Castillo-Chavez (2012), Mathematical Models in Population Biology and Epidemiology, Vol. 2. New York: Springer, 2012.

Brussevich, Mariya, Chris Papageorgiou and Pauline Wibaux (2022), "Trade and the COVID-19 Pandemic: Lessons from French Firms," IMF Working Paper No. 2022/081.

Burlig, Anant Sudarshan, and Garrison Schlauch (2021), "The Impact of Domestic Travel Bans on COVID-19 is Nonlinear in Their Duration ,"NBER Working Paper No. 28699.

Campante, Filipe, and David Yanagizawa-Drott (2018), "Long-Range Growth: Economic Development in the Global Network of Air Links," Quarterly Journal of Economics 133, no. 3 (2018): 1395-1458.

Cerdeiro, Diego A. and Andras Komaromi (2020), "Supply Spillovers During the Pandemic: Evidence from High-Frequency Shipping Data," IMF Working Paper No. 2020/284.

Chaney, Thomas (2014), "The Network Structure of International Trade," American Economic Review 104, no. 11: 3600-3634.

Christakos, George, Ricardo A. Olea, Marc L. Serre, Hwa-Lung Yu and Lin-Lin Wang (2005) Interdisciplinary Public Health Reasoning and Epidemic Modelling: The Case of Black Death, Amsterdam: Springer.

Cristea, Anca D. (2011), "Buyer-Seller Relationships in International Trade: Evidence from U.S. States' Exports and Business-Class Travel," Journal of International Economics 84, no. 2: 207-220.

Cuñat, Alejandro, and Robert Zymek (2020), "The (Structural) Gravity of Epidemics," CESifo Working Paper Series, 8295. 
Debaere, Peter and Hongshik Lee (2004), "The Real-Side Determinants of Countries' Terms of Trade: A Panel Data Analysis," mimeo University of Texas at Austin.

Diamond, Jared (1998) Guns, Germs and Steel: A Short History of Everybody for the Last 13000 Years, London: Vintage.

Diekmann, O., J.A.P. Heesterbeek, J.A.J. Metz (1990), "On the Definition and the Computation of the Basic Reproduction Ratio $R_{0}$ in Models for Infectious Diseases in Heterogeneous Populations," Journal of Mathematical Biology 28, 365-382.

Eaton, Jonathan and Samuel Kortum, (2002), "Technology, Geography, and Trade," Econometrica, 70:5, 1741-1779.

Ellison, Glenn (2020), "Implications of Heterogeneous SIR Models for Analyses of Covid-19," NBER Working Paper No. 27373.

Fauci, Anthony S. (2005) "Emerging and Re-emerging Infectious Diseases: The Perpetual Challenge," , Robert H. Ebert Memorial Lecture, Milbank Memorial Fund.

Fajgelbaum, Pablo D., Amit Khandelwal, Wookun Kim, Cristiano Mantovani, and Edouard Schaal (2021), "Optimal Lockdown in a Commuting Network," American Economic Review: Insights, 3(4), 503-22.

Farboodi, Maryam, Gregor Jarosch, and Robert Shimer (2021), "Internal and External Effects of Social Distancing in a Pandemic," Journal of Economic Theory, 196, 105293.

Fenichel, Eli P., Carlos Castillo-Chavez, M. Graziano Ceddia, Gerardo Chowell, Paula A. Gonzalez Parra, Graham J. Hickling, Garth Holloway et al. (2011), "Adaptive Human Behavior in Epidemiological Models," Proceedings of the National Academy of Sciences 108, no. 15: 6306-6311.

Fernández-Villaverde, Jesús and Chad Jones (2022), "Estimating and Simulating a SIRD Model of Covid-19 for Many Countries, States, and Cities," Journal of Economic Dynamics and Control, 104318.

Garrett, Thomas A. (2009) "War and Pestilence as Labor Market Shocks: US Manufacturing Wage Growth 1914-1919," Economic Inquiry, 47(4), 711-725.

Goolsbee, Austan and Chad Syverson (2021) "Fear, Lockdown, and Diversion: Comparing Drivers of Pandemic Economic Decline 2020," Journal of Public Economics, 104311.

Grépin, Karen Ann, Tsi-Lok Ho, Zhihan Liu, Summer Marion, Julianne Piper, Catherine Z. Worsnop and Kelley Lee (2021) "Evidence of the Effectiveness of Travel-related Measures during the Early Phase of the COVID-19 Pandemic: a Rapid Systematic Review," British Medical Journal Global Health, 6, e004537.

Hethcote, Herbert W. (1978), "An Immunization Model for a Heterogeneous Population," Theoretical Population Biology 14, no. 3: 338-349. 
Hethcote, Herbert W (2000), "The Mathematics of Infectious Diseases," SIAM Review, 42(4), pp.599-653.

Hethcote, H.W. and Thieme, H.R., (1985), "Stability of the Endemic Equilibrium in Epidemic Models with Subpopulations," Mathematical Biosciences, 75(2), pp. 205-227.

Institute of Medicine (2006) Impact of Globalization on Infectious Disease Emergence and Control: Exploring the Consequences and Opportunities, Washington DC: National Academies of Science.

Jedwab, Remi, Noel D. Johnson and Mark Koyama (2019) "Pandemics, Places, and Populations: Evidence from the Black Death," George Washington University, mimeograph.

Jedwab, Remi, Noel D. Johnson and Mark Koyama (2022) "The Economic Impact of the Black Death," Journal of Economic Literature, 60(1), 132-178.

Jones, Callum J, Thomas Philippon, and Venky Venkateswaran (2021), "Optimal Mitigation Policies in a Pandemic: Social Distancing and Working from Home," Review of Financial Studies, 34(11), 5188-5223.

Kang, Seung-Ji, Sooyeon Kim, Kyung-Hwa Park, Sook In Jung, Min-Ho Shin, Sun-Seog Kweon, Hyang Park, Seong-Woo Choi, Eungyu Lee, and So Yeon Ryu (2021) "Successful control of Covid-19 outbreak through tracing, testing, and isolation: Lessons learned from the outbreak control efforts made in a metropolitan city of South Korea," Journal of Infection and Public Health, 14 (9), 1151-1154,

Kennan, J. and J. R. Walker (2011) "The Effect of Expected Income on Individual Migration Decisions," Econometrica, 79(1), 211-251.

Kenny, Charles (2021) The Plague Cycle: The Unending War Between Humanity and Infectious Disease, New York: Scribner.

Kermack, William Ogilvy and A. G. McKendrick, (1927), "A Contribution to the Mathematical Theory of Epidemics, Part I," Proceedings of the Royal Society of London. Series A, 115 (772), pp. 700-721.

Kermack, William Ogilvy and A. G. McKendrick, (1932), "Contributions to the Mathematical Theory of Epidemics. II - The Problem of Endemicity," Proceedings of the Royal Society of London. Series A, 138 (834), pp. 55-83.

Kulendran, N. and Wilson, K. (2000) "Is There a Relationship Between International Trade and International Travel," Applied Economics, 32, 1001-9.

Lafrogne-Joussier, Raphael, Julien Martin and Isabelle Mejean (2021) "Supply shocks in supply chains: Evidence from the early lockdown in China," IMF Economic Review, forthcoming.

Li, Yige, Eduardo A Undurraga, José R. Zubizarreta (2022) "Effectiveness of Localized Lockdowns in the COVID-19 Pandemic," American Journal of Epidemiology, 191(5), 812-824. 
Liu, Xuepeng, Emanuel Ornelas, and Huimin Shi (2022), "The Trade Impact of the COVID-19 Pandemic," The World Economy, forthcoming.

Magal, Pierre, Seydi Ousmane, and Glenn Webb (2016) "Final Size of an Epidemic for a Twogroup SIR Model," Society for Industrial and Applied Mathematics, 76(5), 2042-2059.

Melitz, Marc J. (2003), "The Impact of Trade on Intra-Industry Reallocations and Aggregate Industry Productivity," Econometrica 71, no. 6 (2003): 1695-1725.

Monte, Ferdinando, Stephen J. Redding, and Esteban Rossi-Hansberg. (2018) "Commuting, Migration, and Local Employment Elasticities," American Economic Review, 108(12), 38553890.

Morley, Clive, Jaume Rosselló and Maria Santana-Gallego (2014) "Gravity Models for Tourism Demand: Theory and Use," Annals of Tourism Research, 48, 1-10.

North, Douglass C. and Robert Paul Thomas (1973) The Rise of the Western World: A New Economic History, Cambridge: Cambridge University Press.

Nunn, Nathan and Qian, Nancy (2010) "The Columbian Exchange: A History of Disease, Food and Ideas," Journal of Economic Perspectives, 24(2), 163-188.

Postan, M. M. (1973) Medieval Trade and Finance, Cambridge: Cambridge University Press.

Saker, Lance, Kelley Lee, Barbara Cannito, Anna Gilmore and Diarmid Campbell-Lendrum (2004) "Globalization and Infectious Diseases: A Review of the Linkages," Special Topics in Social, Economic and Behavioural (SEB) Research, Geneva: World Health Organization.

Söderlund (2020) "The Importance of Business Travel for Trade: Evidence from the Liberalization of the Soviet Airspace," Working Paper Series, 1355, Research Institute of Industrial Economics.

Startz, Meredith (2021), "The Value of Face-To-Face: Search and Contracting Problems in Nigerian Trade," mimeo Dartmouth College.

Tatem, A. J., D. J. Rogers and S. I. Hay (2006) "Global Transport Networks and Infectious Disease Spread," Advances in Parasitology, 62, 294-343.

Toxvaerd, Flavio (2020), "Equilibrium Social Distancing," Cambridge-INET Working Paper Series No: $2020 / 08$.

Van den Driessche, Pauline, and James Watmough (2002), "Reproduction Numbers and SubThreshold Endemic Equilibria for Compartmental Models of Disease Transmission," Mathematical Biosciences 180, no. 1-2, pp. 29-48.

Yue, Ricci P. H., Harry F. Lee and Connor Y. H. Wu (2017) "Trade Routes and Plague Transmission in Pre-industrial Europe," Nature, Scientific Reports, 7, 12973, 1-10. 